\title{
A Simultaneous Solution for Reserve Capacity Maximization and Delay Minimization Problems in Signalized Road Networks
}

\author{
Ozgur Baskan $D^{D},{ }^{1}$ Huseyin Ceylan $\left(D,{ }^{1}\right.$ and Cenk Ozan ${ }^{2}$ \\ ${ }^{1}$ Department of Civil Engineering, Faculty of Engineering, Pamukkale University, Denizli 20160, Turkey \\ ${ }^{2}$ Department of Civil Engineering, Faculty of Engineering, Aydin Adnan Menderes University, Aydin 09100, Turkey \\ Correspondence should be addressed to Ozgur Baskan; obaskan@pau.edu.tr
}

Received 14 December 2018; Accepted 25 February 2019; Published 12 May 2019

Academic Editor: Stefano de Luca

Copyright (c) 2019 Ozgur Baskan et al. This is an open access article distributed under the Creative Commons Attribution License, which permits unrestricted use, distribution, and reproduction in any medium, provided the original work is properly cited.

\begin{abstract}
In this study, we present a bilevel programming model in which upper level is defined as a biobjective problem and the lower level is considered as a stochastic user equilibrium assignment problem. It is clear that the biobjective problem has two objectives: the first maximizes the reserve capacity whereas the second minimizes performance index of a road network. We use a weighted-sum method to determine the Pareto optimal solutions of the biobjective problem by applying normalization approach for making the objective functions dimensionless. Following, a differential evolution based heuristic solution algorithm is introduced to overcome the problem presented by use of biobjective bilevel programming model. The first numerical test is conducted on two-junction network in order to represent the effect of the weighting on the solution of combined reserve capacity maximization and delay minimization problem. Allsop \& Charlesworth's network, which is a widely preferred road network in the literature, is selected for the second numerical application in order to present the applicability of the proposed model on a medium-sized signalized road network. Results support authorities who should usually make a choice between two conflicting issues, namely, reserve capacity maximization and delay minimization.
\end{abstract}

\section{Introduction}

As it is well known, road users may be delayed at signalized intersections on urban roads because of implementing inappropriate signal timings even if traffic flow is less than capacity. In order to minimize delay, signal timing optimization concept has widely been used on intersection, arterial or network levels. Implementation of proper signal timing plans on a road network most likely leads to a decrease on flow/capacity ratios and reveals the spare capacity of road segments. This spare capacity can be utilized until the increasing travel demand reaches a certain level. The problem of how to determine this certain level of travel demand, which leads to an flow/capacity ratio equal to one for at least one road segment on the network, has been known as reserve capacity maximization problem (RCMP). The reserve capacity can also be defined as the largest applicable demand multiplier to an existing Origin-Destination (O-D) demand matrix without violating the capacity of any link subject to signal timing parameters and other related constraints. By means of maximizing the reserve capacity of a road network, decision makers reveal the spare capacity and the network becomes more reliable when taking sudden spikes in travel demand into account. Local authorities or agencies that are responsible for traffic operations can benefit from this improvement. On the other hand, road users may suffer from growing travel demand and correspondingly increasing delay. As it is well known, delay function is mainly used as an objective to optimize signal timings for signalized road networks. Although optimal signal timings can be easily found for isolated intersections, signal timing optimization in coordinated road networks becomes highly challenging issue due to the vast search space considering the offset and common cycle time components. Relevant literature provides a large number of studies concerning both reserve capacity maximization and delay minimization on road networks. From the point of RCMP, Webster and Cobbe [1] firstly studied on this problem and they determined the reserve capacity of a signalized intersection by developing an explicit formulation. In order to generalize this approach, Allsop [2] proposed a new method for junctions with complicated 
signal plans. Afterward, Yagar [3, 4] improved the linear program proposed by Allsop [2] considering different saturation flows between successive stages. Wong and Yang [5] applied the reserve capacity approach to a signalized network by considering user equilibrium assignment. They aimed to find the maximum O-D demand multiplier subject to link flows not exceeding their capacities. Yang et al. [6] proposed a bilevel programming model (BLPM) by using combined assignment and trip distribution model to find the network reserve capacity considering equilibrium constraints. Yang and Wang [7] investigated the results of applying two different objectives, namely, reserve capacity maximization and travel cost minimization. It was found that the relationship between these objectives depended on the level of congestion. At the same year, Ziyou and Yifan [8] dealt with the RCMP in a different viewpoint; that is, they considered different demand multipliers between $\mathrm{O}-\mathrm{D}$ pairs to create a condition closer to the reality. Ge et al. [9] investigated whether the reserve capacity was affected by the road users informed about the network or not. Results showed that the reserve capacity did not increase in a similar way with the information level of road users. Ceylan and Bell [10] solved the RCMP by optimizing traffic signal timings including offset term in different stages. They developed a two-stage algorithm in the stochastic user equilibrium (SUE) manner. Chen et al. [11] proposed an approach considering both reliability and capacity to solve the RCMP. For this purpose, they developed a new index to determine the demand multiplier by using a BLPM. Chiou [12] solved the RCMP based on a projected gradient method. The proposed method was compared with traditional methods and results showed that it outperformed other methods by means of reducing delay.

As good examples in terms of biobjective problems, Chiou [13, 14] presented novel algorithms for the solution of the RCMP with toll settings. Encouraging results were attained from two studies through the provided numerical examples. Similarly, Chiou [15] introduced a hybrid approach to maximize the reserve capacity and to minimize delay based on link capacity expansions. Another type of biobjective problems has been tackled with minimizing delay and maximizing reserve capacity with time-invariant flows by Chiou [16]. From a different viewpoint, Miandoabchi and Farahani [17] solved the RCMP in the context of discrete network design problem using a bilevel solution approach. In a similar manner, Miandoabchi et al. [18] handled this combined problem related to road network design to optimize network reserve capacity and some related performance measures. Chiou [19] defined the reserve capacity maximization and delay minimization problems as a min-max problem subject to the equilibrium constraints. Proposed model was applied to two signal controlled benchmark networks, which have widely been used in studies concerning network design problem, and results showed that the model was capable of solving the RCMP by taking delay minimization problem into account. Wang et al. [20] solved the RCMP with SUE link flows and subsequently extended it by considering maximum link capacity expansions with limited budget. Xiao et al. [21] investigated the RCMP from a different viewpoint, and they developed a zone-based model to reveal the effects of using maximum capacity on the land-use development. Recently, Han and Cheng [22] studied on the effectiveness of the tradable credit scheme, which is a particular type of congestion pricing applications, by considering the RCMP with SUE link flows. To this end, they developed a bilevel model which was applied to two road networks to illustrate the positive effect of the tradable credit scheme. Baskan and Ozan [23] aimed to maximize the reserve capacity by considering equity issue with deterministic user equilibrium link flows. They presented a bilevel heuristic solution algorithm based on Harmony Search (HS) technique. Results indicated that the value of O-D demand multiplier was highly sensitive to the equity parameter. Recently, Li and Sun [24] proposed a novel optimization method for signal control in urban road networks by drawing attention to the lack of studies on multiobjective optimization. Road networks with different scales were used in order to reveal the applicability of the proposed method by considering network throughput, delay, and spillbacks. Results showed that the proposed multiobjective optimization method was quite promising.

From the point of delay minimization problem, TRANSYT-7F traffic software, which consists of a traffic model and a signal timing optimizer, is nearly most preferred tool for signalized networks (see for details McTrans Center [25]) in order to optimize signal timings. It simulates traffic in a signalized road network to produce flow profiles which are then used to determine a performance index $(P I)$ by considering specific stage plans and signal timing parameters. The $P I$, which is used to define the cost of traffic congestion in a road network, is defined as the sum of a weighted combination of delay and number of stops. In this context, several studies have been presented in the literature. As known, Webster [26] presented one of the first studies using mathematical framework in order to minimize delay on an isolated junction. This study was further extended by Allsop [27] to develop a more general approach in which total delay was minimized by formulating signal setting problem as a convex mathematical programming. After these first attempts to optimize traffic signal timings, Wong [28] carried perspective on signal timing optimization a step further and developed approximate mathematical expressions for the derivatives of $P I$ by considering phase-based control variables. It was concluded from this study that the use of approximate expressions provided benefit in terms of computational effort. A year later, Heydecker [29] applied decomposition approach to optimize traffic signal timings for road networks. Application of this method to a small example network showed significant benefits in terms of network performance. Wong [30] used group-based control variables to optimize signal timings with fixed link flows. For this purpose, the PI was considered as a function of group-based control variables. To overcome some drawbacks in the study by Wong [30] in terms of computational effort, parallel computing technique was presented to reduce computational time by Wong [31]. In Wong et al. [32], TRANSYT traffic model was converted to a timedependent model to determine PI. Girianna and Benekohal [33] developed two different heuristic algorithms based on Genetic Algorithm (GA) for oversaturated signalized 
networks. Results indicated that micro-GA method was able to reach quickly to the near-global signal timings in comparison with the traditional GA. Ceylan and Bell [34] introduced a GA-based model to optimize signal timings with SUE link flows. They employed TRANSYT software to calculate performance index of a road network whereas SUE link flows were calculated using path flow estimator. Similarly, Ceylan and Bell [35], a year later, used the same GAbased model to reveal its capability in finding optimal signal timings under congestion with different demand conditions. Ceylan [36] proposed a GA-based model using TRANSYT Hill-Climbing (HC) optimization tool for area traffic control. The proposed algorithm outperformed TRANSYT in terms of performance index although it had some deficiencies regarding computational burden due to the use of $\mathrm{HC}$ optimization tool. Chen and $\mathrm{Xu}$ [37] used Particle Swarm Optimization (PSO) algorithm in order to find optimal signal timings. Their results revealed that the proposed algorithm was applicable especially in different demand conditions. Dan and Xiaohong [38] investigated the use of improved version of the GA to solve signal timing problem by taking the offset term into account and they reached valuable results in terms of minimizing delay and improving the network capacity. Li [39] developed an optimization model considering an arterial road to find optimal signal settings under oversaturated conditions. Moreover, developed model was able to take complex flow interaction between lanes into account with the cell transmission concept. Liu and Chang [40] presented a new signal optimization model considering mutual interaction between queues occurred on different lanes on arterial links. Results showed that their model outperformed TRANSYT-7F for planning arterial signals. Ceylan and Ceylan [41] developed a hybrid algorithm integrating HS and HC optimization methods. In order to find optimal signal settings, the performance index was determined by TRANSYT traffic software. Results revealed that the proposed algorithm outperformed conventional HS and GA methods in terms of PI. Dell'Orco et al. [42] presented a HS-based method using BLPM for signalized road networks by considering SUE link flows. Similarly, Dell'Orco et al. [43] attempted to solve area traffic control problem by using Artificial Bee Colony (ABC) algorithm with TRANSYT-7F. Results revealed that the $\mathrm{ABC}$ algorithm outperformed the GA and HC methods in terms of PI. Zhu et al. [44] developed Reinforcement Learning (RL) based Junction Tree Algorithm for solving signal control problem in which agents present traffic signals. Results proved that the proposed algorithm was able to produce better results than other RL-based methods. Ozan et al. [45] developed a modified RL algorithm to optimize traffic signal timings for road networks. Results showed that the proposed algorithm was capable of finding optimal signal parameters even in the case of increasing demand conditions. Christofa et al. [46] presented a realtime signal control system aiming to minimize person delay by optimizing signal timings based on mixed integer linear programming approach. Field tests proved that the person based minimization approach outperforms fixed-time signal timings. Zhang et al. [47] developed two novel models in order to solve signal coordination problem especially for long arterials and grid networks. Proposed models were in the form of a mixed-integer linear program. Numerical experiments revealed that both models were able to produce consistent coordination plans in terms of travel speed, stop, and delay. Srivastava and Sahana [48] proposed a hybrid evolutionary approach to optimize signal timings using BLPM. Comparative experiments showed that the hybrid model outperforms Ant Colony Optimization (ACO) and GA techniques. Recently, Memoli et al. [49] focused on signal setting design at network-level by introducing a stage-based method. In proposed method, stage sequences, stage lengths, and offsets were optimized simultaneously. In order to show the applicability of the proposed model, two example test networks have been analyzed. Results indicated that the developed method was an effective way to design network signal settings.

In the relevant literature, reserve capacity maximization or delay minimization has been mostly performed by optimizing traffic signal timings. However, to the best of our knowledge, there is no study in which they are simultaneously considered. Although Ceylan and Bell [10] presented a twostage approach to the solution of the RCMP by taking delay minimization into account, their study had a limitation, that is, the signal timings were optimized in one stage by minimizing PI. The largest O-D matrix multiplier was found in the second stage subject to the best signal timings found in the first stage. This means that the biobjective BLPM was separately solved in their study. Therefore, we define a new approach for investigating the advantage of the simultaneous solution of the RCMP and delay minimization problem which are both considered as a biobjective problem. To evaluate the users' reaction to this arrangement performed at the upper level, the lower level is presented as a SUE assignment problem. In this context, it is assumed that the local authority tries to simultaneously optimize traffic signal timings by maximizing the reserve capacity and minimizing PI by taking into account the users' reactions in SUE manner. This mutual interaction between users and local authority is presented by using biobjective BLPM. In summary, the major contributions to the current literature made in this paper are as follows. (i) A biobjective BLPM is proposed. The upper level maximizes the reserve capacity of a road network and minimizes PI. The lower level solves SUE problem by using the path flow estimator (PFE) which follows logit route choice model. (ii) A weighted-sum method is applied to find Pareto optimal solutions of the biobjective problem by normalizing the objective functions. (iii) A biobjective BLPM is applied to a medium-sized signalized road network.

The rest of this paper is organized as follows. The next section is about the problem formulation. Section 3 presents detailed explanations of the proposed b-objective BLPM. The numerical experiments are given in Section 4. The conclusions and future directions are given in the last section.

\section{Problem Formulation}

Considering that the local authorities or agencies are responsible for traffic planning, data collection, sign and signal operations, etc., they desire robust road networks in terms 
of congestion and delay even in case of sudden spikes in travel demand. On the other hand, traffic flow is distributed on a road network based on route choice behaviour of users that aim to complete their travels within the shortest possible travel time. Therefore, maximizing reserve capacity and minimizing delay provide reasonable benefits for local authorities and road users, respectively. However, maximizing reserve capacity may reduce network performance by increasing delay because of growing travel demand. In this study, this trade-off is investigated by defining a biobjective BLPM. At the upper level of the proposed model, combined reserve capacity maximization and delay minimization problem is solved, while SUE assignment problem is performed using $\mathrm{PFE}$ at the lower level. Considering a road network with a set of O-D pairs, $K$, a set of directed links, $A$, a set of paths, $P$, and a set of nodes, $N$, the proposed biobjective BLPM is presented as follows:

$$
\begin{array}{ll}
\max _{\psi, \mathbf{x}^{*}} & \mu \\
\min _{\psi, \mathbf{x}^{*}} & P I=\sum_{a \in A}\left[w_{a}^{d} \cdot d_{a}(\psi)+K \cdot w_{a}^{s} \cdot S_{a}(\psi)\right]
\end{array}
$$

subject to

$$
\begin{aligned}
& \psi(c, \boldsymbol{\theta}, \boldsymbol{\varphi}) \in \boldsymbol{\Omega}_{0} ;\left\{\begin{array}{l}
c_{\min } \leq c \leq c_{\max } \\
0 \leq \theta<c \\
\varphi_{\min } \leq \varphi \leq c \\
z \\
\sum_{i=1}(\varphi+I)_{i}=c
\end{array}\right. \\
& x_{a}^{*}(\mu, \psi) \leq C_{a}\left(\psi, s_{a}\right)
\end{aligned}
$$

where (1) represents the problem of reserve capacity maximization and (2) is related to delay minimization problem. In addition, $\mu$ is the demand matrix multiplier, $d_{a}$ is delay on link $a, a \in A, S_{a}$ is the number of stops on link $a, w_{a}^{d}$ and $w_{a}^{s}$ are weighting factors for $d_{a}$ and for $S_{a}$ on link $a$, respectively, $K$ is stop penalty factor, $\mathbf{x}^{*}$ is vector of equilibrium link flows, $c$ is common cycle time, $c_{\min }$ and $c_{\max }$ are possible bounds for common cycle time, $\varphi$ is stage green time, $\varphi_{\min }$ is the minimum green time, $I$ is intergreen time, $\theta$ is offset, $\psi$ is vector of signal timings, $\boldsymbol{\Omega}_{0}$ is feasible region for signal timings, and $z$ is the number of stages. In addition to signal timings related constraints, (4) represents link capacity constraint in which $s_{a}$ is the saturation flow, $C_{a}$ is the capacity, and $x_{a}^{*}(\mu, \psi)$ is equilibrium flow on link $a$. Link flows can be obtained by solving SUE problem using (5)-(6) at the lower level [50].

$$
\begin{aligned}
& \min _{\mathbf{x}(\psi)} F(\mathbf{x}(\psi), \psi) \\
&=- \mu \mathbf{q}^{\mathrm{T}} \mathbf{y}(\mathbf{x}(\psi), \psi)+\mathbf{x}^{\mathrm{T}} \mathbf{t}(\mathbf{x}(\psi), \psi) \\
&-\sum_{a \in A} \int_{0}^{x_{a}(\psi)} t_{a}(\psi, w) d w
\end{aligned}
$$

subject to

$$
\mu \mathbf{q}=\Lambda \mathbf{h},
$$

$$
\begin{aligned}
& \mathbf{x}(\boldsymbol{\psi})=\boldsymbol{\delta} \mathbf{h}, \\
& \mathbf{h} \geq \mathbf{0}
\end{aligned}
$$

where $\mathbf{q}$ is the vector of travel demand, $\mathbf{t}$ and $\mathbf{y}$ represent vectors of link and path travel times for the given vector of link flows $\mathbf{x}(\psi)$, respectively, $\mathbf{h}$ is the vector of path flows, $\boldsymbol{\delta}$ represents the link/path incidence matrix where $\delta_{a p}=1$ if link $a$ is on path $p$, and $\delta_{a p}=0$ otherwise $\left[\delta_{a p} ; \forall a \in A ; \forall p \in\right.$ $P]$, and $\Lambda$ is the $\mathrm{O}-\mathrm{D} /$ path incidence matrix $\left[\Lambda_{p} ; \forall p \in P\right]$. The SUE problem given in (5) can be solved by the PFE [51]. The PFE is a logit based traffic assignment tool and its most important advantage is that it does not require path enumeration. Therefore it is most preferable option for researches for the solution of SUE assignment [34, 35, 41, 50].

Since there are two objectives in the upper level in (1)-(4), which are mutually interacted, it is not easy to find optimal solution between two objectives. Thus, a suitable solution has to be found among Pareto optimal solutions. Although there are several efficient methods to find the set of Pareto optimal solutions of biobjective problems, the weighted-sum method can be used as an efficient tool to convert a biobjective problem into single-objective by using convex linear combination of objectives [52]. On the other hand, since each singleobjective problem (delay minimization and reserve capacity maximization) has a different unit, they cannot be directly added. To overcome this issue, they can be normalized before applying the weighted-sum method as given in

$$
\begin{aligned}
& Z_{1}=\frac{\mu^{*}}{\mu} \\
& Z_{2}=\frac{P I}{P I^{*}}
\end{aligned}
$$

where $\mu^{*}$ can be stated as an upper limit of reserve capacity maximization problem which is the solution of the singleobjective problem given in (1) subject to (3)-(6). On the other hand, $P I^{*}$ is the lower limit of delay minimization problem and can be found by solving (2) subject to (3) and (5)-(6). It should be emphasized that the use of $Z_{1}$ converts the reserve capacity maximization problem into a minimization problem. Thus, objective functions $Z_{1}$ and $Z_{2}$ can be stated as minimization problems. After normalizing the objective functions, they are considered as scalar, and thus there is no further obstacle for applying the weighted-sum method to convert the biobjective problem into a single-objective problem as given in (9) subject to (3)-(6).

$$
\min Z\left(\mu, \mathbf{x}^{*}, \psi\right)=\alpha Z_{1}+(1-\alpha) Z_{2}
$$

For applying weighted-sum method we have used a weighting factor $\alpha$ in order to present relative importance of one objective to other. In other words, the value of $\alpha$ is specified to find the balance between solutions of network reserve capacity maximization and delay minimization problems. It should be noted that a larger value of $\alpha$ implies that the local authority should be more attentive to the improvement of network reserve capacity. On the other side, the authority prioritizes to minimize network overall delay 


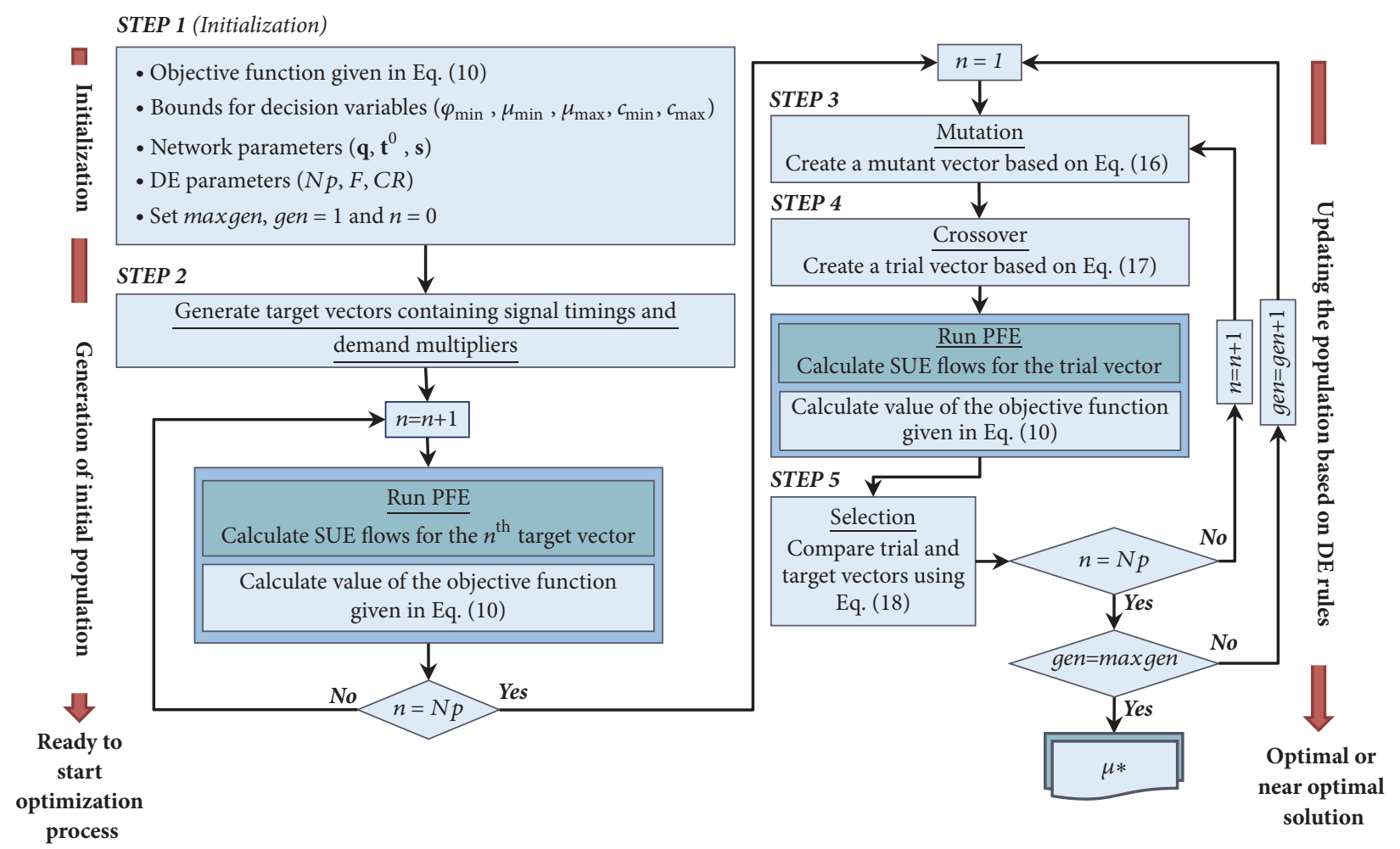

FIGURE 1: The reserve capacity maximization model.

in comparison with reserve capacity maximization when a smaller value of $\alpha$ is used. Choosing a suitable value of weighting factor can be performed by generating a uniform set of weights as $\alpha=\{0,1 / m, 2 / m, \ldots . .(m-1) / m, 1\}$, where $m$ is a positive integer number [53].

\section{Model Development}

In this study, the proposed biobjective BLPM is developed based on differential evolution (DE) optimization framework. As known, DE is referred as a robust and simple metaheuristic algorithm proposed by [54]. On the other hand, several studies in the relevant literature support this claim by introducing the advantages of DE in solving different complex optimization problems. Nolle et al. [55] have made a comparison of self-organizing migration algorithm (SOMA) with simulated annealing (SA) and DE algorithms for automated waveform tuning. Although a slight difference has been observed between performances of DE and SOMA algorithms, they produced similar computational performance in this application outperforming SA. In the study by Vincenzi et al. [56] the performance of DE algorithm has been compared with Coupled Local Minimizers (CLM) for solving dynamic damage detection problem. Results revealed that DE outperforms CLM method especially in case the number of decision variables is increased. Similarly, a comparison of DE algorithm with superior metaheuristic algorithms has been performed on different benchmark functions by Civicioglu and Besdok [57]. According to the results, DE produces more robust results compared with other metaheuristic algorithms.
Deb et al. [58] have compared the performances of DE, PSO, and GA in solving design problem of circularly polarized microstrip antennas. DE based algorithm outperformed GA and PSO according to the numerical applications. Recently, Dezelak et al. [59] tested the performances of DE and PSO algorithms for power plants modelling. Results have shown that DE produces better results in reasonable computation time than PSO. In the light of short review of DE algorithm, it has been chosen for solving the biobjective BLPM by considering its successful applications.

3.1. Reserve Capacity Maximization Model. As previously mentioned, values of the maximum O-D multiplier, $\mu^{*}$, and the minimum network performance index, $P I^{*}$, are required to set up the single-objective model given in (9) for obtaining the Pareto optimal solutions of the biobjective optimization problem. In this context, both problems are handled with a BLPM based on the DE solution framework to obtain $\mu^{*}$ and $P I^{*}$, in which the network O-D multiplier and $P I$ are optimized at the upper level. On the other hand, SUE assignment problem is solved to determine equilibrium link flows at the lower levels of both models. In this context, maximization of the O-D multiplier is formulated as a minimization problem subject to (3)-(6) as follows:

$$
\begin{aligned}
& \min u\left(\mu, \mathbf{x}^{*}, \psi\right) \\
& =\frac{1}{\mu}+\sigma\left[\sum_{a \in A} \max \left(x_{a}^{*}(\mu, \psi)-C_{a}\left(\psi, s_{a}\right), 0\right)\right]
\end{aligned}
$$

where $\sigma$ is a constant penalty weighting factor. Five-step solution framework is illustrated in Figure 1. 
Step 1. The objective function representing the maximization of network reserve capacity given in (10) and the possible bounds for decision variables, which are common cycle time, stage green times, and O-D multiplier, are primarily initialized. Subsequently, network parameters, namely, saturation flows, free-flow travel times, and travel demand, are represented. Initialization of three DE algorithm parameters is also carried out at this step. The first one is population size, $N p$, and the next one is mutation factor, $F$, which is used to create mutant vector. Crossover rate $(C R)$ is the third parameter, which is employed in creating the trial vector [54]. At the end of this step, generation number (gen) is set to 1 , iteration number $(n)$ is set to 0 , and maximum number of generations (maxgen) is identified as the stopping criterion.

Step 2. At this step, initial solution vectors $\Gamma$ including $\mathrm{O}$ $\mathrm{D}$ multipliers and signal timings are generated considering preset bounds of decision variables. Subsequently, objective function values are calculated and represented as given in

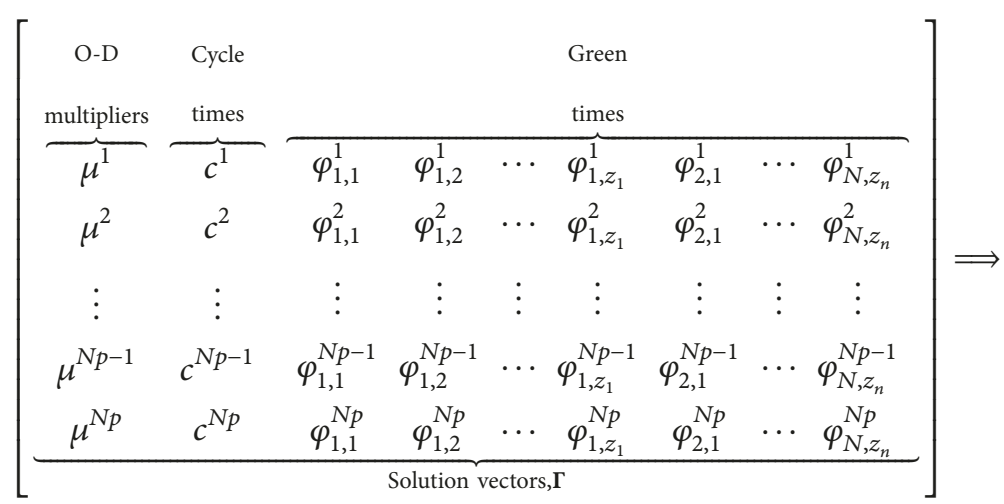

$$
\left[\begin{array}{c}
\text { Objective } \\
\frac{u\left(\mathbf{x}^{*}(\boldsymbol{\Gamma}), \Gamma\right)^{1}}{u\left(\mathbf{x}^{*}(\boldsymbol{\Gamma}), \boldsymbol{\Gamma}\right)^{2}} \\
\cdots \\
u\left(\mathbf{x}^{*}(\boldsymbol{\Gamma}), \Gamma\right)^{N p-1} \\
u\left(\mathbf{x}^{*}(\boldsymbol{\Gamma}), \Gamma\right)^{N p}
\end{array}\right]
$$

where $\varphi_{i, j}$ is the $j^{\text {th }}$ stage green time of intersection $i$ and $z_{n}$ is the number of stages at $n^{\text {th }}$ intersection $(i=1,2, \ldots, N$ and $\left.j=1,2, \ldots, z_{n}\right)$. In this study, initial values of the O-D multipliers and signal timing parameters are obtained in the following way.

(i) $O-D$ multiplier is generated between 0 and possible upper bound as given in

$$
\mu=\operatorname{rand}(0,1] \times \mu_{\max }
$$

(ii) Cycle time is randomly generated between the possible bounds as given in

$$
c=\operatorname{int}\left[\operatorname{rand}[0,1] \times\left(c_{\max }-c_{\min }\right)+c_{\min }\right]
$$

(iii) Stage green times at intersections are generated between $\varphi_{\min }$ and $c$ as shown in

$$
\varphi_{i, j}=\operatorname{int}\left[\operatorname{rand}[0,1] \times\left(c-\varphi_{\min }\right)+\varphi_{\min }\right]
$$

Subsequently, generated green timing values are revised since all green plus intergreen times must be equal to the cycle time for a given intersection. This procedure is carried out by

$$
\begin{array}{r}
\varphi_{i, j}=\varphi_{\min }+\frac{\varphi_{i, j}}{\sum_{j=1}^{z_{i}} \varphi_{i, j}}\left[c-z_{i} \times\left(I+\varphi_{\min }\right)\right] \\
\\
\quad i=1,2, \ldots z
\end{array}
$$

As can be seen in (11) the equilibrium link flows, $\mathbf{x}^{*}$, are required to calculate the objective function values. In this context, the SUE assignment problem, which was given in (5), is solved by the PFE traffic assignment tool, the pseudo code of which is given in Algorithm 1 .

In Algorithm 1, $\beta$ represents dispersion parameter. Note that if $\beta$ is zero then the path costs are not fully taken into account in path choice which means even highest cost paths can be chosen by road users. On the contrary, drivers become sensitive to cost in path choice while $\beta$ tends to infinity.

Step 3. The mutation is carried out at this step by using three different, randomly chosen solution vectors which are 
different from target vector (i.e., solution vector) and a mutant vector, $\boldsymbol{\rho}_{i}$, is created as given in

$$
\boldsymbol{\rho}_{i, g e n}=\boldsymbol{\Gamma}_{r 0, \text { gen }}+F \cdot\left(\boldsymbol{\Gamma}_{r 1, \text { gen }}-\boldsymbol{\Gamma}_{r 2, \text { gen }}\right)
$$

where $r 0, r 1$, and $r 2$ are indices of randomly chosen solution vectors.

Step 4. At this step, crossover is applied by choosing each member of the trial vector, $\mathbf{E}_{i}$, from the target or the mutant vectors with the probabilities of $C R$ or $1-C R$, respectively, as given in

$$
\begin{aligned}
\mathbf{E}_{i, \text { gen }} & =\varepsilon_{j, i, g e n} \\
& = \begin{cases}\rho_{j, i, g e n} & \text { if }\left(\text { rand }_{j}(0,1) \leq C R \text { or } j=j_{\text {rand }}\right) \\
\gamma_{j, i, g e n} & \text { otherwise }\end{cases}
\end{aligned}
$$

where the condition of $j=j_{\text {rand }}$ provides that target and trial vectors are definitely different from each other. After determining the members of the trial vector, their related SUE link flows and the objective function value are calculated.

Step 5. At the last step, the target vector, $\boldsymbol{\Gamma}_{i, g e n+1}$, for the next generation is selected by comparing the objective function values of trial and target vectors as given in

$$
\begin{aligned}
& \boldsymbol{\Gamma}_{i, \text { gen }+1} \\
& = \begin{cases}\mathbf{E}_{i, \text { gen }} & \text { if } u\left(\mathbf{x}^{*}\left(\mathbf{E}_{i, \text { gen }}\right), \mathbf{E}_{i, \text { gen }}\right) \leq u\left(\mathbf{x}^{*}\left(\boldsymbol{\Gamma}_{i, g e n}\right), \boldsymbol{\Gamma}_{i, g e n}\right) \\
\boldsymbol{\Gamma}_{i, \text { gen }} & \text { otherwise }\end{cases}
\end{aligned}
$$

The DE process is repeated until the maximum number of generations, maxgen, is reached.

3.2. Delay Minimization Model. Following the representation of the reserve capacity maximization model, flowchart of the delay minimization model for the solution of (2) is illustrated in Figure 2.

Step 1. At this step, the objective function given in (2) subject to (3) and (5)-(6) representing the minimization of network, $P I$, and the possible bounds for signal timing variables are primarily initialized. Note that, unlike the reserve capacity maximization model, O-D multiplier is set to 1 , which means that the base travel demand is loaded into the network. The remaining of this step is the same as the first step of the reserve capacity maximization model.

Step 2. Initial signal timings are generated between preset bounds based on (13)-(15). Moreover, the offset variables are randomly generated as shown in

$$
\theta_{i}=\operatorname{int}[\operatorname{rand}[0,1) * c]
$$

As can be seen in (2) the equilibrium link flows are required to calculate network $P I$ value. Thus, the SUE assignment problem, which was given in (5), is solved by the PFE for each solution vector in the population pool. Subsequently, TRANSYT-7F is employed to calculate the related PI values and the target vectors, namely, the initial solutions, are stored in the memory as given in

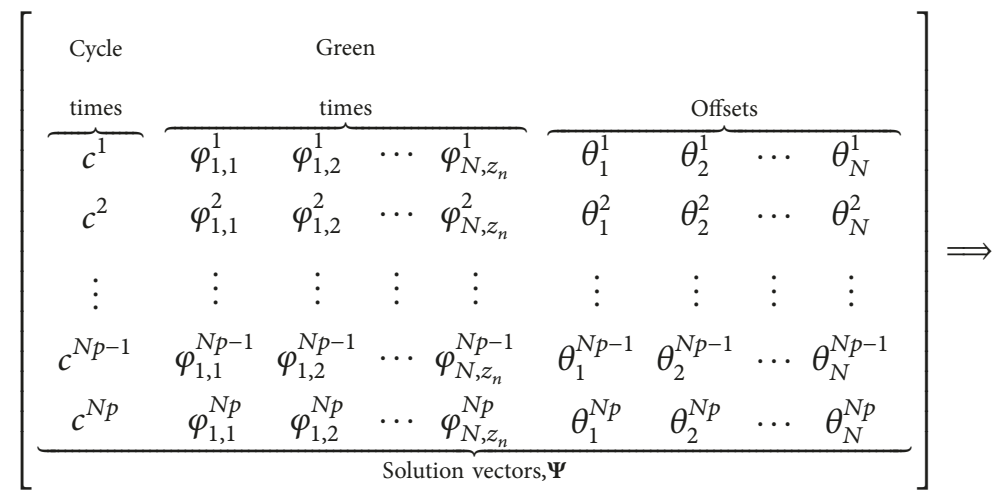

$$
\left[\begin{array}{c}
\text { Objective } \\
\frac{\text { functions }}{P I\left(\mathbf{x}^{*}(\boldsymbol{\Psi}), \boldsymbol{\Psi}\right)^{1}} \\
P I\left(\mathbf{x}^{*}(\Psi), \Psi\right)^{2} \\
\cdots \\
P I\left(\mathbf{x}^{*}(\Psi), \Psi\right)^{N p-1} \\
P I\left(\mathbf{x}^{*}(\Psi), \Psi\right)^{N p}
\end{array}\right]
$$




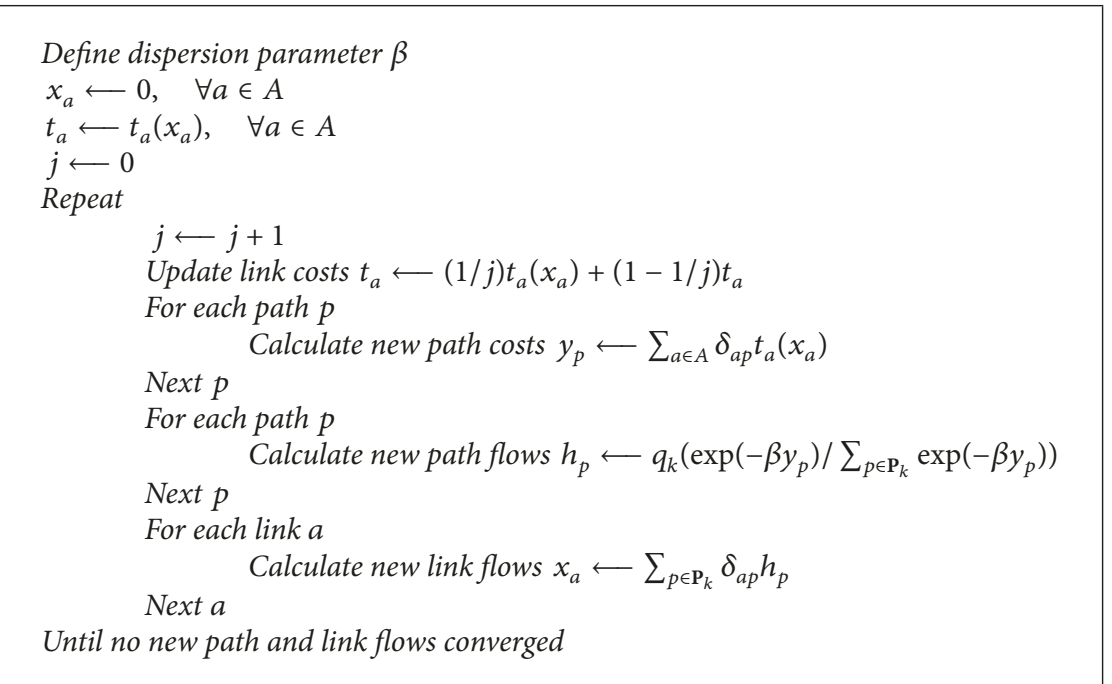

Algorithm 1: Pseudo code of the PFE.
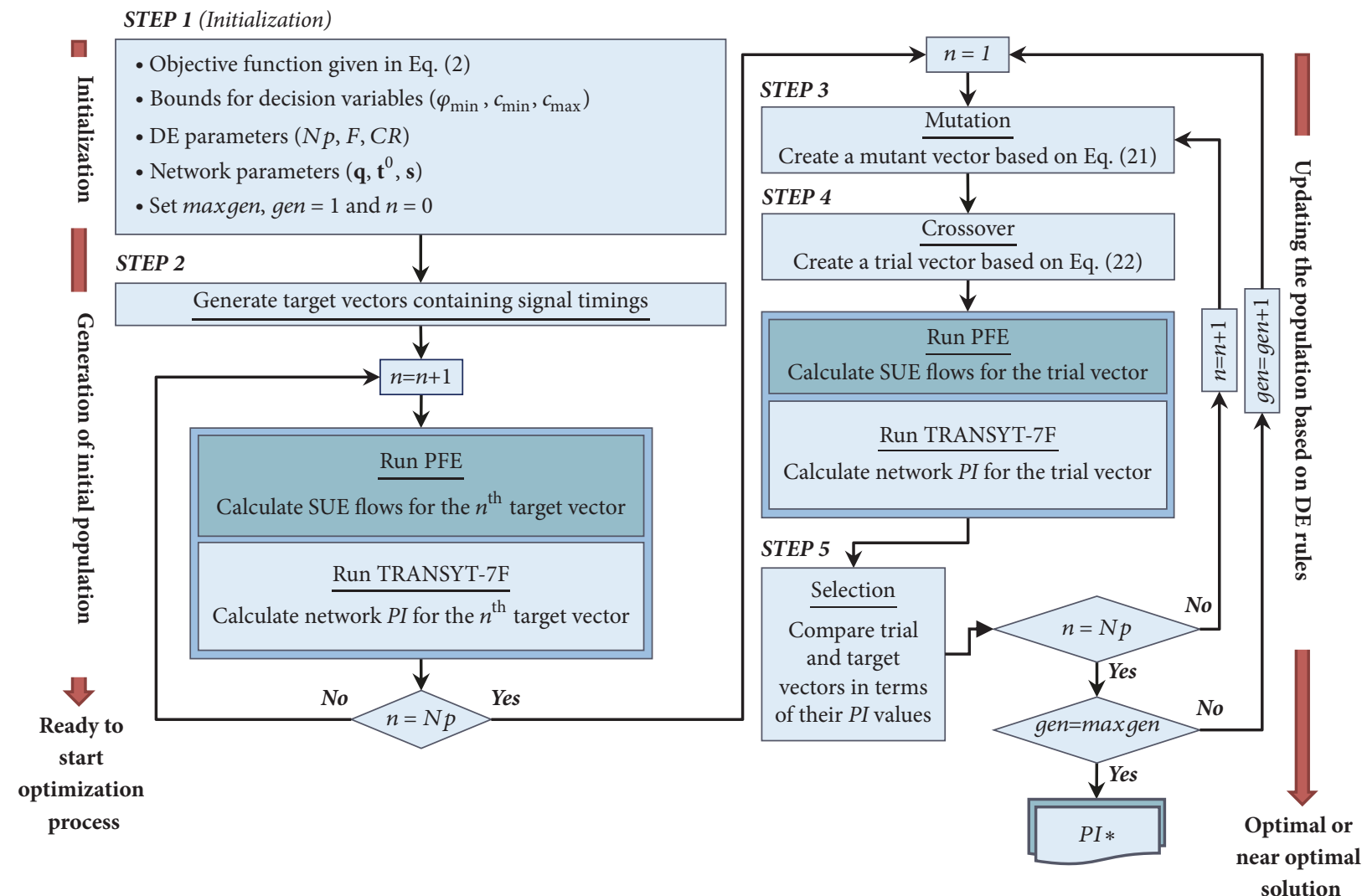

Figure 2: The delay minimization model.

Step 3. The mutation is carried out to create a mutant vector, $\boldsymbol{\rho}_{i}$, which is given in

$$
\boldsymbol{\rho}_{i, \text { gen }}=\Psi_{r 0, \text { gen }}+F \cdot\left(\Psi_{r 1, \text { gen }}-\Psi_{r 2, \text { gen }}\right)
$$

Step 4. At this step, crossover is applied to determine the members of the trial vector, $\mathbf{E}_{i}$, as given in

$$
\mathbf{E}_{i, g e n}=\varepsilon_{j, i, g e n}
$$

$$
= \begin{cases}\rho_{j, i, g e n} & \text { if }\left(\operatorname{rand}_{j}(0,1) \leq C R \text { or } j=j_{\text {rand }}\right) \\ \psi_{j, i, g e n} & \text { otherwise }\end{cases}
$$

Step 5. Selection is carried out in terms of the objective function values of trial vector, $\mathbf{E}_{i, g e n}$, and its target vector, $\Psi_{i, \text { gen }}$, in the following way. 


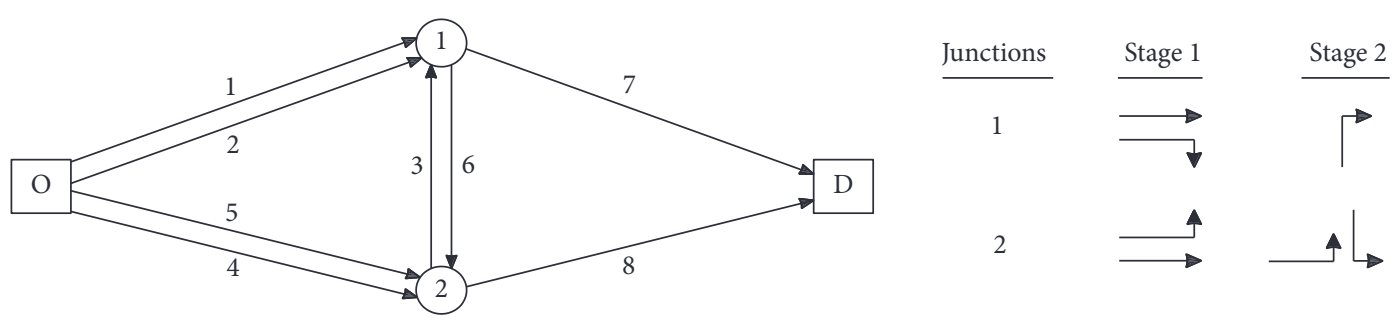

FIGURE 3: Layout of two-junction network and representation of signal stages.

TABLE 1: Input data for two-junction network.

\begin{tabular}{lccccccc}
\hline Junction number & Link number & $t_{a}^{0}(\mathrm{~s})$ & $s_{a}(\mathrm{veh} / \mathrm{hr})$ & Junction number & Link number & $t_{a}^{0}(\mathrm{~s})$ & $s_{a}(\mathrm{veh} / \mathrm{hr})$ \\
\hline 1 & 1 & 20 & 1800 & 2 & 4 & 20 & 1800 \\
& 2 & 20 & 1800 & & 5 & 20 & 1800 \\
& 3 & 20 & 1800 & 6 & 20 & 1800 \\
\hline
\end{tabular}

where $t_{a}^{0}$ is free flow travel time in seconds on link $a$.

$$
\begin{aligned}
& \boldsymbol{\Psi}_{i, \text { gen }+1} \\
& = \begin{cases}\mathbf{E}_{i, g e n} & \text { if } P I\left(\mathbf{x}^{*}\left(\mathbf{E}_{i, g e n}\right), \mathbf{E}_{i, g e n}\right) \leq P I\left(\mathbf{x}^{*}\left(\boldsymbol{\Psi}_{i, g e n}\right), \Psi_{i, g e n}\right) \\
\boldsymbol{\Psi}_{i, g e n} & \text { otherwise }\end{cases}
\end{aligned}
$$

The DE process is repeated until the maximum number of generations, maxgen, is reached.

3.3. Combined Model. Once the values of $\mu^{*}$ and $P I^{*}$ are obtained, the combined reserve capacity maximization and delay minimization problem is solved using single-objective BLPM in (9) subject to (3)-(6) by taking a particular weighting factor $\alpha$ into account. The corresponding stepwise procedure is given below.

Step 1. Generate the initial signal timing vectors, $\Psi$, and $\mathrm{O}-$ $\mathrm{D}$ multipliers, $\mu$, with given possible bounds and calculate objective function values, which is given in (9), as many as population size.

Step 2. Set gen $=1$ and $n=0$.

Step 3. Set $n=n+1$. Then, generate a new signal timing vector, $\Psi$, and a new $\mathrm{O}-\mathrm{D}$ multiplier, $\mu$, based on mutation and crossover operations of DE.

Step 4. Calculate $Z_{1}$ based on the O-D multiplier, $\mu$, which has been generated in the previous step, and $\mu^{*}$ as given in (7).

Step 5. Run PFE to carry out a SUE assignment for estimating link flows based on the O-D multiplier, $\mu$, and the signal timing vector, $\Psi$, which have been generated in Step 3 . Subsequently, run TRANSYT-7F to obtain the network PI value and then calculate $Z_{2}$ based on $P I$ and $P I^{*}$ as given in (8).

Step 6. Calculate objective function $Z$ as given in (9).

Step 7. Compare the $n^{\text {th }}$ solution vector in the population pool and newly generated solution vector in terms of their objective function values. Subsequently, keep the one with a better objective function value and discard the other.

Step 8. Go to Step 3 if $n \neq N p$.

Step 9. Set $n=0$ and gen $=$ gen +1 and then go to Step 3 if gen $\neq$ maxgen .

Step 10. Terminate the DE process and output the optimized signal timing vector and O-D multiplier.

\section{Numerical Applications}

In this section, two signalized benchmark road networks are chosen to investigate the performance of the proposed biobjective BLPM. Firstly, a two-junction network is used to show the effect of weighting on the balance of solution of reserve capacity maximization and delay minimization problems. In other words, it is proposed to find the set of Pareto optimal solutions of the biobjective problem. With this application, it is also aimed at investigating how the network performance is affected by applying the biobjective BLPM depending upon the weighting factor $\alpha$. Secondly, the effectiveness of the proposed model is investigated on a medium-sized road network presented by Allsop and Charlesworth [60].

4.1. Two-Junction Network. In order to test the capability of the proposed model in solving the biobjective problem, it has been firstly applied to the two-junction network taken from Ceylan [61]. Layout of the network and the input data are given in Figure 3 and Table 1, respectively. Travel demand between the single O-D pair is taken as $1500 \mathrm{veh} / \mathrm{hr}$.

The intergreen time, $I$, between stages was selected as $5 \mathrm{sec}$. Constraints for signal timing variables are set as given below:

$$
\psi(c, \boldsymbol{\theta}, \boldsymbol{\varphi}) \in \mathbf{\Omega}_{0} ;\left\{\begin{array}{l}
30 \leq c \leq 100 \\
0 \leq \theta<c \\
7 \leq \varphi \leq c
\end{array}\right.
$$


TABLE 2: Network PI values and corresponding standard deviations for each parameter combination.

\begin{tabular}{|c|c|c|c|c|c|}
\hline$F$ & $C R$ & Best $P I\left(P I^{*}\right)$ & Mean $P I$ & Worst $P I$ & Standard dev. \\
\hline 0.5 & 0.8 & 7.06 & 7.16 & 7.34 & 0.11 \\
\hline 0.5 & 0.9 & 7.06 & 7.19 & 7.43 & 0.14 \\
\hline 0.5 & 1.0 & 7.09 & 7.21 & 7.34 & 0.12 \\
\hline 0.6 & 0.8 & 7.02 & 7.12 & 7.34 & 0.10 \\
\hline 0.6 & 0.9 & 7.02 & 7.12 & 7.21 & 0.08 \\
\hline 0.6 & 1.0 & 7.09 & 7.18 & 7.34 & 0.08 \\
\hline 0.7 & 0.8 & 7.02 & 7.10 & 7.34 & 0.10 \\
\hline 0.7 & 0.9 & 7.02 & 7.14 & 7.34 & 0.10 \\
\hline 0.7 & 1.0 & 7.09 & 7.18 & 7.52 & 0.15 \\
\hline 0.8 & 0.8 & 7.02 & 7.06 & 7.09 & 0.04 \\
\hline 0.8 & 0.9 & 7.02 & 7.11 & 7.34 & 0.13 \\
\hline 0.8 & 1.0 & 7.09 & 7.16 & 7.34 & 0.09 \\
\hline 0.9 & 0.8 & 7.02 & 7.09 & 7.21 & 0.07 \\
\hline 0.9 & 0.9 & 7.09 & 7.22 & 7.34 & 0.13 \\
\hline 0.9 & 1.0 & 7.21 & 7.32 & 7.52 & 0.15 \\
\hline 1.0 & 0.8 & 7.04 & 7.18 & 7.34 & 0.09 \\
\hline 1.0 & 0.9 & 7.21 & 7.29 & 7.34 & 0.07 \\
\hline 1.0 & 1.0 & 7.34 & 7.39 & 7.52 & 0.09 \\
\hline
\end{tabular}

TABLE 3: Flow parameters for $P I^{*}$ at two-junction network.

\begin{tabular}{|c|c|c|c|}
\hline Link number & Capacity (veh/hr) & Flow (veh/hr) & Degree of saturation (\%) \\
\hline 1 & 1332 & 751 & 56 \\
\hline 2 & 1332 & 0 & 0 \\
\hline 3 & 288 & 0 & 0 \\
\hline 4 & 1260 & 749 & 59 \\
\hline 5 & 360 & 0 & 0 \\
\hline 6 & 360 & 0 & 0 \\
\hline
\end{tabular}

TABLE 4: Signal timings for $P I^{*}$ at two-junction network.

\begin{tabular}{|c|c|c|c|c|}
\hline \multirow[b]{2}{*}{$\begin{array}{l}\text { Cycle time } \\
c(\mathrm{~s})\end{array}$} & \multirow{2}{*}{$\begin{array}{c}\text { Junction } \\
\text { number } \\
j\end{array}$} & \multicolumn{2}{|c|}{ Duration of stage green timings (s) } & \multirow{2}{*}{$\begin{array}{c}\text { Offsets }(\mathrm{s}) \\
\theta_{j}\end{array}$} \\
\hline & & $\begin{array}{c}\text { Stage 1 } \\
\varphi_{j, 1}\end{array}$ & $\begin{array}{c}\text { Stage } 2 \\
\varphi_{j, 2}\end{array}$ & \\
\hline \multirow{2}{*}{100} & 1 & 79 & 21 & 0 \\
\hline & 2 & 75 & 25 & 38 \\
\hline
\end{tabular}

Setting of mutation factor $F$, crossover rate $C R$, and population size $N p$ is crucial for the performance of DE algorithm. Storn and Price [54] recommend that the ranges of $[0.5,1.0]$ and $[0.8,1.0]$ may be chosen for $F$ and $C R$, respectively. In this context, an analysis is performed for 18 parameter combinations where $F$ and $C R$ are varied from 0.5 to 1.0 and from 0.8 to 1.0 in steps of 0.1 , respectively. 30 independent runs are performed for every parameter combination for solving delay minimization problem and the corresponding results are given in Table $2 . N p$ is set as 20 based on the recommendation given in Rönkkönen et al. [62] and maxgen is set as 100 . Note that the average computational time for complete run of the proposed model is about 28 minutes. It means that each generation takes about 16.8 seconds of CPU. The proposed model has been executed in MATLAB programming and performed on PC with Intel Core i7 2.10 GHz, RAM 8 GB.

It can be seen in Table 2 that $P I^{*}$ value is obtained as 7.02 with 7 different parameter combinations. In addition to that, the setting $F=C R=0.8$ resulted in the lowest mean $P I$ and standard deviation values which are 7.06 and 0.04 , respectively. Therefore, this parameter combination is selected for further applications. Corresponding results for $P I^{*}$ at two-junction network are presented in Tables 3 and 4. As can be seen in Table 3, the demand was almost equally shared by links 1 and 4 . Other links in the network do not carry flow according to the solution for $P I^{*}$.

Once the network $P I^{*}$ has been calculated, the reserve capacity maximization problem can be solved for twojunction network assuming that the bounds for $\mathrm{O}-\mathrm{D}$ 
TABLE 5: Flow parameters for $\mu^{*}$ at two-junction network.

\begin{tabular}{lccc}
\hline Link number & Capacity (veh/hr) & Flow (veh/hr) & Degree of saturation (\%) \\
\hline 1 & 810 & 810 & 100 \\
2 & 810 & 794 & 98 \\
3 & 810 & 793 & 98 \\
4 & 810 & 810 & 100 \\
5 & 810 & 793 & 98 \\
6 & 810 & 794 & 98 \\
\hline
\end{tabular}

TABLE 6: Signal timings for $\mu^{*}$ at two-junction network.

\begin{tabular}{|c|c|c|c|}
\hline \multirow{3}{*}{$\begin{array}{l}\text { Cycle Time } \\
c(s)\end{array}$} & \multirow{3}{*}{$\begin{array}{c}\text { Junction } \\
\text { number } \\
j\end{array}$} & \multicolumn{2}{|c|}{ Duration of stage green timings (s) } \\
\hline & & Stage 1 & Stage 2 \\
\hline & & $\varphi_{j, 1}$ & $\varphi_{j, 2}$ \\
\hline \multirow{2}{*}{100} & 1 & 50 & 50 \\
\hline & 2 & 50 & 50 \\
\hline
\end{tabular}

TABLE 7: Values of $Z, Z_{1}$ and $Z_{2}$ at two-junction network.

\begin{tabular}{lccc}
\hline$\alpha$ & $Z_{1}$ & $Z_{2}$ & $Z$ \\
\hline 0.00 & 2.137 & 1.000 & 1.000 \\
0.10 & 2.097 & 1.023 & 1.130 \\
0.20 & 2.057 & 1.045 & 1.247 \\
0.30 & 2.019 & 1.069 & 1.354 \\
0.40 & 1.981 & 1.092 & 1.448 \\
0.50 & 1.908 & 1.153 & 1.530 \\
0.60 & 1.834 & 1.391 & 1.657 \\
0.70 & 1.691 & 1.752 & 1.709 \\
0.80 & 1.549 & 2.217 & 1.683 \\
0.90 & 1.330 & 4.840 & 1.681 \\
1.00 & 1.000 & 14.240 & 1.000 \\
\hline
\end{tabular}

multiplier are $(0,5)$. According to the analysis, $\mu^{*}$ was found as 2.1370 which is the solution of the single-objective problem given in (10). Other corresponding results obtained with the solution are given in Tables 5 and 6, respectively.

As shown in Table 5, the values of degree of saturation on the links indicate that none of the links is oversaturated although the O-D demand was multiplied by 2.1370 . In other words, flows do not exceed their corresponding link capacities. In the case that O-D demand matrix was multiplied by $\mu^{*}$, the cycle time was found as 100 which is its upper bound for this example. This means that the common cycle time was increased, together with the O-D multiplier to overcome the increased demand which was more than double of the base demand on the network.

Once the values of $\mu^{*}$ and $P I^{*}$ are obtained, there is no further obstacle to solve the combined reserve capacity maximization and delay minimization problem by using (9). Optimized values of the objective functions, $Z, Z_{1}$, and $Z_{2}$, are given for different values of weighting factors in Table 7. It can be seen in Table 7 that, in case $\alpha$ is zero, any improvement in the network reserve capacity is not allowed. Thus, the value of $Z_{1}$ becomes equal to $\mu^{*}$ since $\mu$ is 1.0 which represents the

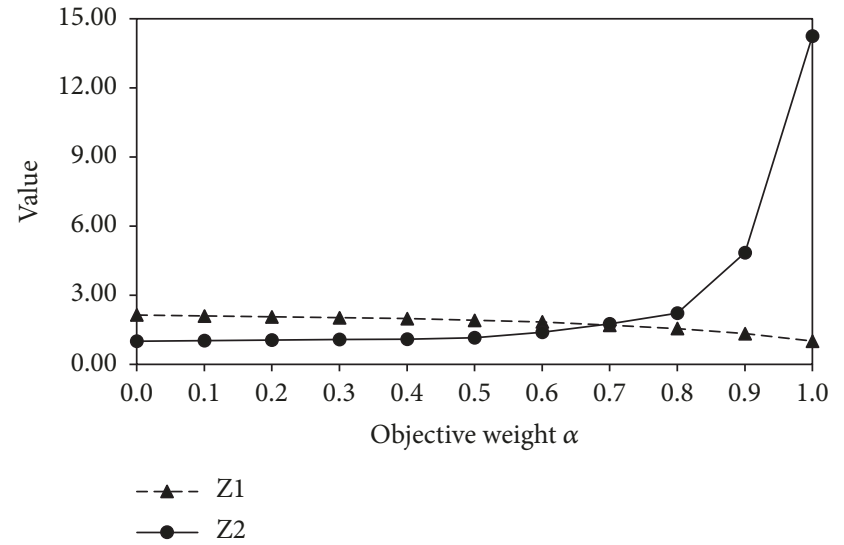

FIgURE 4: Variation of $Z_{1}$ and $Z_{2}$ for different $\alpha$ values.

base demand. On the other hand, the value of $Z_{2}$ equals 1.0 indicating that the optimized value of $P I$ is equivalent to $P I^{*}$ which is the upper bound of PI. In brief, the authority is fully concerned with the delay minimization and no additional travel demand is added to the network. On the contrary, when $\alpha$ equals 1.0, the authority is not further concerned with the delay minimization and tries to maximize the reserve capacity in the network and consequently the value of $Z_{1}$ equals 1.0 which means that $\mu$ becomes equal to $\mu^{*}$.

In addition, the graphical representation for the values of $Z_{1}$ and $Z_{2}$ regarding different values of $\alpha$ is given in Figure 4 which also shows the Pareto optimal solutions for the objective functions, $Z_{1}$ and $Z_{2}$. It can be emphasized that $Z_{1}$ and $Z_{2}$ are in conflict with each other; that is, $Z_{1}$ shows a decreasing tendency while $Z_{2}$ has a tendency to increase. Thus, regarding the dilemma between reserve capacity maximization and delay minimization problems, the authority can choose one among the Pareto optimal solutions according to its own preferences. It can be seen that there is no significant change in the values of $Z_{1}$ and $Z_{2}$ when the value of $\alpha$ increases from 0 to 0.5 . Afterwards, the value of $Z_{2}$ tends to increase in case $\alpha$ is higher than 0.5. Likewise, it can be clearly observed that the value of $Z_{2}$ dramatically increases when $\alpha$ is higher than 0.8 . This indicates that the authority pays more attention to the network reserve capacity maximization than delay minimization.

Depending on the variation of $\alpha$ values between 0 and 1 , values of $\mu$ and $P I$, their changes, and cumulative changes are given in Table 8 . As can be seen in Table $8 \mu$ and PI increase about $8 \%$ and $9 \%$, respectively, while the objective weight 
TABLE 8: Evaluation of the changes of $\mu$ and PI for different $\alpha$ values.

\begin{tabular}{|c|c|c|c|c|c|c|c|}
\hline \multirow{2}{*}{$\begin{array}{l}\text { Objective } \\
\text { weighting } \\
\text { factor } \\
\alpha^{i}\end{array}$} & \multirow{2}{*}{$\begin{array}{c}\text { O-D demand } \\
\text { multiplier } \\
\mu_{\alpha^{i}}\end{array}$} & \multirow{2}{*}{$\begin{array}{c}\text { Network } \\
\text { Performance } \\
\text { index } \\
P I_{\alpha^{i}}\end{array}$} & \multicolumn{2}{|c|}{ Change (\%) } & \multicolumn{2}{|c|}{ Cumulative change (\%) } & \multirow{2}{*}{$\begin{array}{l}\begin{array}{l}\text { Cumulative } \\
\text { change rate }\end{array} \\
\frac{\sum \Delta P I}{\sum \Delta \mu}\end{array}$} \\
\hline & & & $\Delta \mu=\frac{\mu_{\alpha^{i}}-\mu_{\alpha^{i-1}}}{\mu_{\alpha^{i-1}}}$ & $\Delta P I=\frac{P I_{\alpha^{i}}-P I_{\alpha^{i-1}}}{P I_{\alpha^{i-1}}}$ & $\sum \Delta \mu$ & $\sum \Delta P I$ & \\
\hline 0.00 & 1.000 & 7.02 & 1.91 & 2.25 & 1.91 & 2.25 & 1.18 \\
\hline 0.10 & 1.019 & 7.18 & 1.95 & 2.20 & 3.86 & 4.45 & 1.15 \\
\hline 0.20 & 1.039 & 7.34 & 1.87 & 2.30 & 5.73 & 6.75 & 1.18 \\
\hline 0.30 & 1.058 & 7.50 & 1.91 & 2.15 & 7.64 & 8.90 & 1.16 \\
\hline 0.40 & 1.079 & 7.67 & 3.86 & 5.59 & 11.50 & 14.48 & 1.26 \\
\hline 0.50 & 1.120 & 8.09 & 4.01 & 20.64 & 15.51 & 35.13 & 2.26 \\
\hline 0.60 & 1.165 & 9.76 & 8.46 & 25.95 & 23.97 & 61.08 & 2.55 \\
\hline 0.70 & 1.264 & 12.30 & 9.17 & 26.54 & 33.14 & 87.62 & 2.64 \\
\hline 0.80 & 1.380 & 15.56 & 16.47 & 118.31 & 49.60 & 205.93 & 4.15 \\
\hline 0.90 & 1.607 & 33.98 & 33.00 & 194.21 & 82.60 & 400.15 & 4.84 \\
\hline 1.00 & 2.137 & 99.96 & & & & & \\
\hline
\end{tabular}

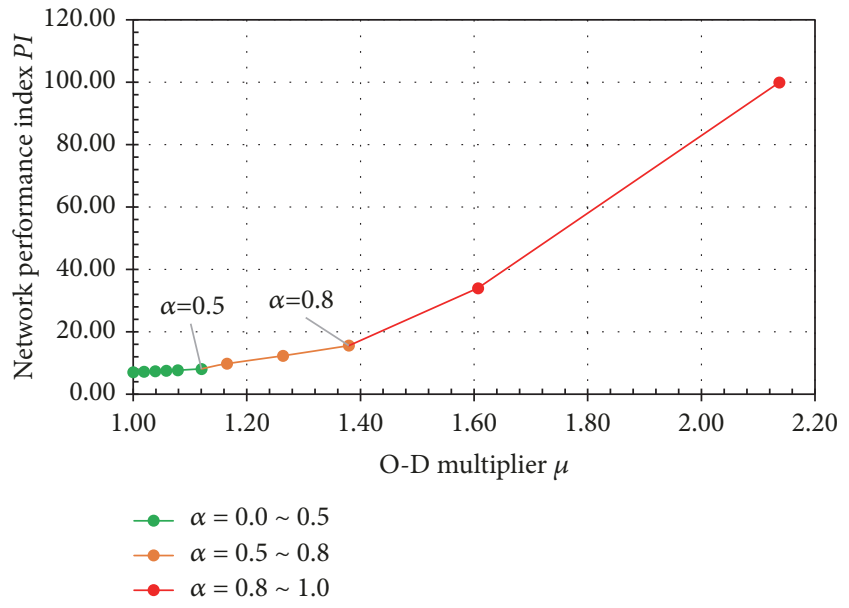

FIgURE 5: Change of PI values with O-D multiplier $\mu$.

increases from 0 to 0.4 . That is, approximately $2 \%$ change is observed for both $\mu$ and PI for every 0.1 change in $\alpha$. However, when $\alpha=0.5$ is selected as the solution point, change in $\mu$ and $P I$ is realized to be about $4 \%$ and $6 \%$, respectively. This means that the users' benefit is badly affected by growing travel demand. Examining the rest of Table 8, it can be seen that there are two significant breaking points in terms of the changes in $\mu$ and PI. The first one is $\alpha=0.6$; that is, the increases in $\mu$ and $P I$ are about $4 \%$ and $21 \%$, respectively. The second point is $\alpha=0.9$; that is, $\mu$ and $P I$ increases are about $16 \%$ and $118 \%$, respectively. This result can be clearly seen in Figure 5 which represents the change of PI with the O-D multiplier $\mu$.

For further explanation of the changes of $\mu$ and $P I$, we present Figure 6 in which their cumulative changes and corresponding change rates are considered with the objective weight of $\alpha$. Bars with three different colours in Figure 6, which represent the cumulative change rates $\sum \Delta P I / \sum \Delta \mu$,

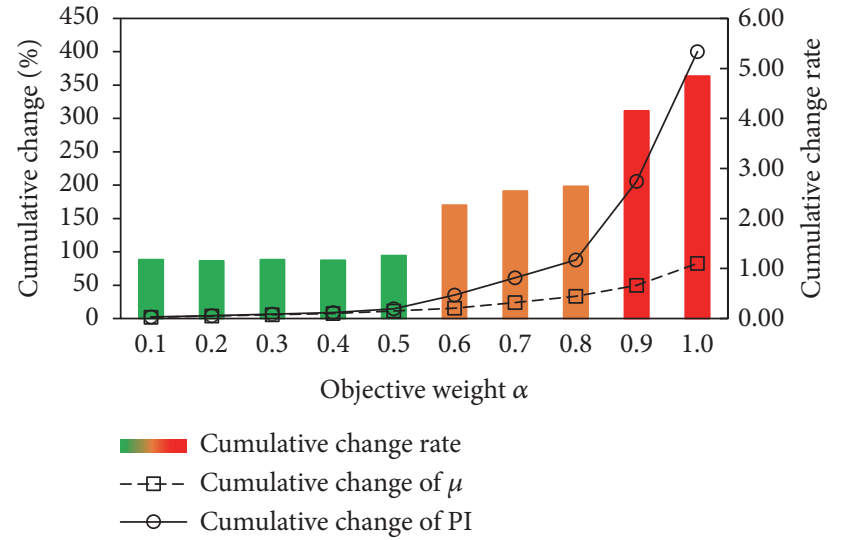

Figure 6: Cumulative changes of $\mu$ and $P I$ with the objective weight of $\alpha$.

reveal three different zones where each one has specific values of cumulative change rates. Therefore, the local authority can make decision about $\alpha$ by considering the first points of those zones. Two breaking points, which are $\alpha=0.6$ and $\alpha=0.9$ as explained based on Table 8 , are clearly seen in the figure. When $\alpha$ equals 0.9 , a more significant increase in cumulative $P I$ value is observed than that for $\alpha=0.6$. Therefore, $\alpha=0.8$ may be considered as the best objective weight that provides an O-D multiplier and signal timing configuration since all other Pareto optimal solutions proportionally reduce users' benefit more than the gain in network reserve capacity.

4.2. Allsop \& Charlesworth's Network. Allsop \& Charlesworth's test network is chosen as second numerical example since it has widely been used in the studies concerning transportation network design [35, 36, 41, 42, 45]. Thus, we aim to give readers a chance to compare some results drawn by some previous studies. The representation of the test road network and its stage plans are given in Figure 7. The example 


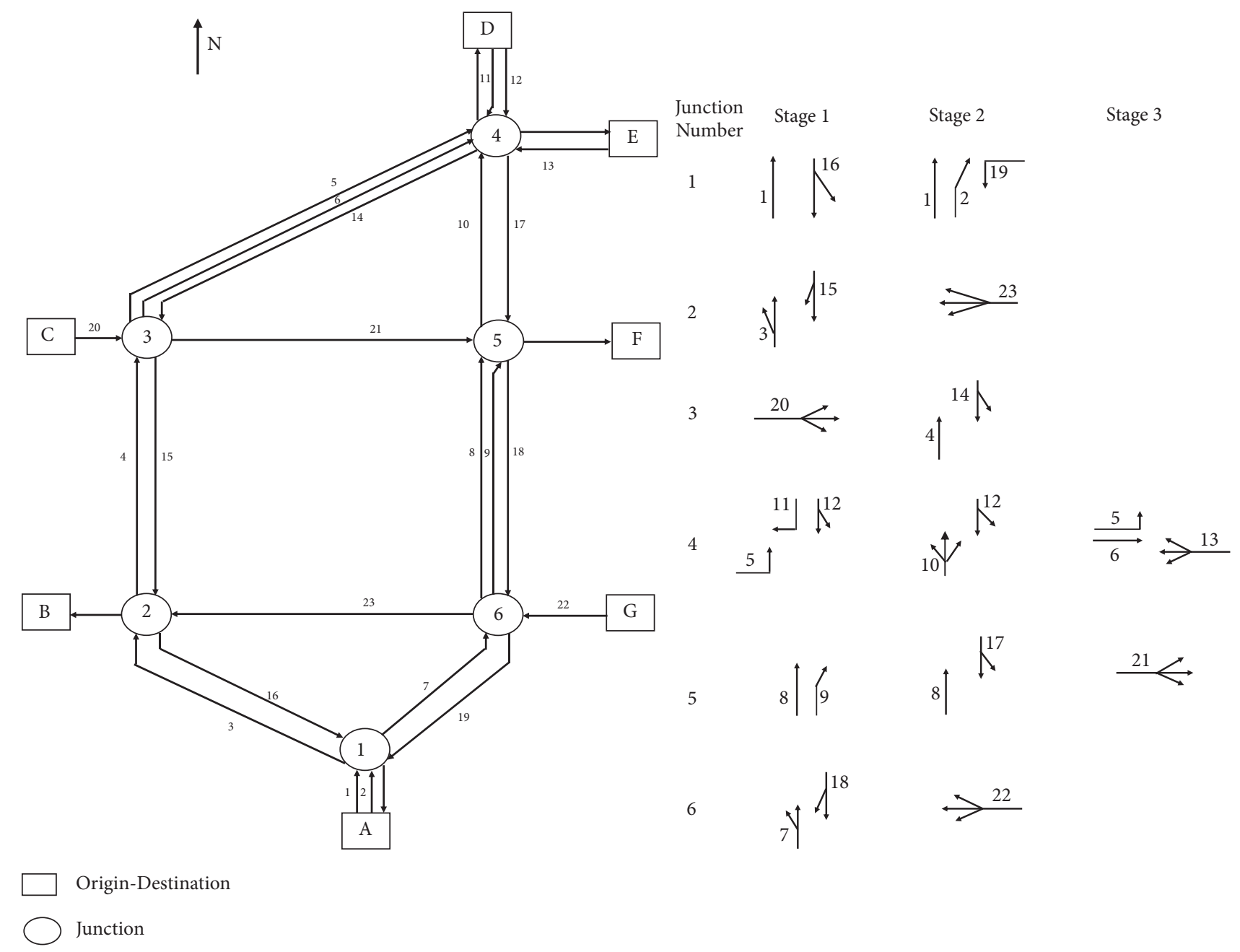

FIgURE 7: Layout and stage plans of Allsop \& Charlesworth's network.

network consists of 23 links and 21 signal setting variables at six junctions. The input data and relevant O-D demand matrix are given in Tables 9 and 10, respectively. below:

Constraints for each signal timing variable are set as given

$$
\psi(c, \boldsymbol{\theta}, \boldsymbol{\varphi}) \in \boldsymbol{\Omega}_{0} ;\left\{\begin{array}{l}
30 \leq c \leq 150 \\
0 \leq \theta<c \\
7 \leq \varphi \leq c
\end{array}\right.
$$

The intergreen time, $I$, between stages was selected as $5 \mathrm{sec}$. In addition, bounds for O-D multiplier were assumed to be $(0,3)$. It should be pointed out that the reason for selecting these upper and lower bounds for O-D multiplier on this example is to reduce the feasible search space and thus to decrease the computational burden of proposed solution algorithm. Considering the number of decision variables for Allsop \& Charlesworth's network, $N p$ and maxgen are selected as 40 and 500, respectively. Firstly, the RCMP was solved by using BLPM given in Figure 1 , and $\mu^{*}$ was found as 1.29 for Allsop \& Charlesworth's network which is the solution of the single-objective problem given in (10).
Subsequently, $P I^{*}$ was determined as 341.10 by solving the delay minimization problem by using the proposed model given in Figure 2. Similarly to the Pareto efficiency analysis performed for two-junction network, the best values of $\mu$ and $P I$ with their changes and cumulative changes after 10 independent runs are given in Table 11 for $\alpha$ values between 0 and 1 . The average computational time for complete run of the proposed model is about 4.7 hours. As can be seen in Table 11, $\mu$ and $P I$ increased on average by $1.0 \%$ and $4.8 \%$, respectively, for every 0.1 change in $\alpha$ while it increases from 0 to 0.8 . On the other hand, while $\alpha$ equals 0.9 , the changes of $\mu$ and PI are about 6 and 8 times higher than their values in the case for $\alpha=0.8$. This means that the users' benefit is badly affected by significant increase in network PI. Therefore, $\alpha=0.8$ may be considered as the best objective weight that provides a balance between solutions of reserve capacity maximization and delay minimization problems. This result can be seen in Figure 8 which represents the change of PI with the O-D multiplier $\mu$.

Resulting SUE link flows, degrees of saturations, and signal timings for $\alpha$ values of $0.0,0.8$, and 1.0 are given in Tables 12 and 13 , respectively. 
TABLE 9: Input data for Allsop \& Charlesworth's network.

\begin{tabular}{|c|c|c|c|c|c|c|c|}
\hline Junction & Link & $\begin{array}{l}\text { Free-flow } \\
\text { travel time } \\
\quad\left(t_{a}^{0}\right)\end{array}$ & $\begin{array}{c}\text { Saturation } \\
\text { flow }\left(s_{a}\right)\end{array}$ & Junction & Link & Free-flow travel time $\left(t_{a}^{0}\right)$ & Saturation flow $\left(s_{a}\right)$ \\
\hline \multirow{6}{*}{1} & & & & \multirow{6}{*}{4} & 5 & 20 & 1800 \\
\hline & 1 & 1 & 2000 & & 6 & 20 & 1850 \\
\hline & 2 & 1 & 1600 & & 10 & 10 & 2200 \\
\hline & 16 & 10 & 2900 & & 11 & 1 & 2000 \\
\hline & 19 & 10 & 1500 & & 12 & 1 & 1800 \\
\hline & & & & & 13 & 1 & 2200 \\
\hline \multirow{4}{*}{2} & & & & \multirow{4}{*}{5} & 8 & 15 & 1850 \\
\hline & 3 & 10 & 3200 & & 9 & 15 & 1700 \\
\hline & $\begin{array}{l}15 \\
23\end{array}$ & $\begin{array}{l}15 \\
15\end{array}$ & $\begin{array}{l}2600 \\
3200\end{array}$ & & 17 & 10 & 1700 \\
\hline & & & & & 21 & 15 & 3200 \\
\hline \multirow{3}{*}{3} & 4 & 15 & 3200 & \multirow{3}{*}{6} & 7 & 10 & 1800 \\
\hline & 14 & 20 & 3200 & & 18 & 15 & 1700 \\
\hline & 20 & 1 & 2800 & & 22 & 1 & 3600 \\
\hline
\end{tabular}

TABLE 10: O-D demand matrix for Allsop \& Charlesworth's network in veh/hr.

\begin{tabular}{lccccc}
\hline O/D & A & B & D & E & F \\
\hline A & -- & 250 & 700 & 30 & 200 \\
C & 40 & 20 & 200 & 130 & 900 \\
D & 400 & 250 & -- & 0 & 100 \\
E & 300 & 130 & 0 & -- & 20 \\
G & 550 & 450 & 170 & 60 & 20 \\
\hline
\end{tabular}

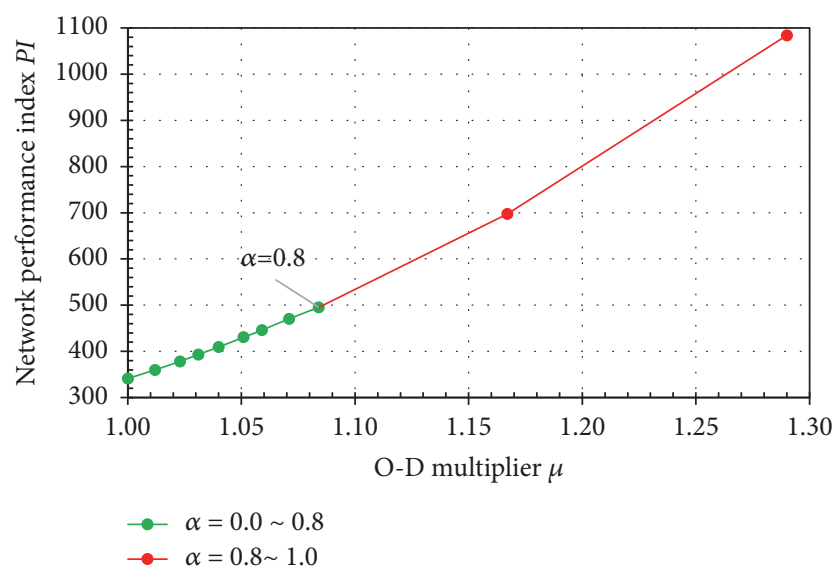

Figure 8: Change of PI values with O-D multiplier $\mu$ for Allsop and Charlesworth's network.

When the value of $\alpha$ is 0 , that is, only the delay minimization is taken into account by the authority, the degrees of saturation of SUE links converge to the lowest values as shown in Table 12. On the contrary, in case of $\alpha=1.0$, which represents that the authority is totally concerned with the maximizing of reserve capacity, $P I$ has substantially increased about 3.2 times, namely, 1084 for the maximum O-D multiplier of 1.29 in comparison with the case of $\alpha=0$ as shown in Table 13. Moreover, although the O-D demand matrix is increased about $30 \%$, none of the links is oversaturated which means that their degrees of saturation are less than 1.0. For $\alpha=0.8, \mu$ and $P I$ are found as 1.08 and 495.40 , respectively. It means that when $\alpha$ equals 0.8 , the road network can accommodate about $8 \%$ more travel demand with ensuring links do not exceed their capacities while PI increases about $45 \%$. This is because $\alpha$ equals 0.8 and the authority does not pay attention to the delay minimization as much as the maximization of reserve capacity problem. On the other side, the common cycle time for Allsop \& Charlesworth's network was found as 150, 138, and 150 sec for $\alpha$ values of $0,0.8$, and 1.0 , respectively.

\section{Conclusions and Future Studies}

This study deals with the simultaneous solution of reserve capacity maximization and delay minimization problems by optimizing traffic signal timings. For this purpose, a biobjective BLPM is developed and solved by implementing a DE based heuristic solution algorithm. We introduce the weighted-sum method to solve the proposed biobjective model and present an approach to make the objective functions of the biobjective model dimensionless. In the upper level, it is aimed to maximize reserve capacity and to minimize delay in a road network, whereas at the lower level the user's reactions are determined by solving stochastic traffic assignment problem.

Two numerical applications are performed in order to show the applicability of the proposed model. Firstly, the proposed model is applied to a two-junction network. In this application, the cumulative change of $P I$ value is extremely high when $\alpha$ is set to 1.0. It means that the authority is totally concerned with the reserve capacity maximization without taking delay minimization into account. Thus, the PI value spikes up to about 100. For the two-junction network, $\alpha=0.8$ is selected as a suitable objective weight that 
TABLE 11: Evaluation of the changes of $\mu$ and PI for different $\alpha$ values for Allsop and Charlesworth's network.

\begin{tabular}{|c|c|c|c|c|c|c|c|}
\hline \multirow{2}{*}{$\begin{array}{l}\text { Objective } \\
\text { weighting } \\
\text { factor } \\
\alpha^{i}\end{array}$} & \multirow{2}{*}{$\begin{array}{c}\text { O-D demand } \\
\text { multiplier } \\
\mu_{\alpha^{i}}\end{array}$} & \multirow{2}{*}{$\begin{array}{c}\text { Network } \\
\text { performance } \\
\text { index } \\
P I_{\alpha^{i}}\end{array}$} & \multicolumn{2}{|c|}{ Change (\%) } & \multicolumn{2}{|c|}{ Cumulative change (\%) } & \multirow{2}{*}{$\begin{array}{l}\begin{array}{l}\text { Cumulative } \\
\text { change rate }\end{array} \\
\frac{\sum \Delta P I}{\sum \Delta \mu}\end{array}$} \\
\hline & & & $\Delta \mu=\frac{\mu_{\alpha^{i}}-\mu_{\alpha^{i-1}}}{\mu_{\alpha^{i-1}}}$ & $\Delta P I=\frac{P I_{\alpha^{i}}-P I_{\alpha^{i-1}}}{P I_{\alpha^{i-1}}}$ & $\sum \Delta \mu$ & $\sum \Delta P I$ & \\
\hline 0.00 & 1.000 & 341.10 & 1.20 & 5.53 & 1.20 & 5.53 & 4.61 \\
\hline 0.10 & 1.012 & 359.97 & 1.09 & 5.13 & 2.29 & 10.66 & 4.64 \\
\hline 0.20 & 1.023 & 378.43 & 0.78 & 3.88 & 3.07 & 14.54 & 4.74 \\
\hline 0.30 & 1.031 & 393.13 & 0.87 & 4.17 & 3.94 & 18.71 & 4.75 \\
\hline 0.40 & 1.040 & 409.51 & 1.06 & 5.16 & 5.00 & 23.87 & 4.77 \\
\hline 0.50 & 1.051 & 430.63 & 0.76 & 3.58 & 5.76 & 27.45 & 4.76 \\
\hline 0.60 & 1.059 & 446.04 & 1.13 & 5.54 & 6.89 & 32.98 & 4.78 \\
\hline 0.70 & 1.071 & 470.73 & 1.21 & 5.24 & 8.11 & 38.22 & 4.71 \\
\hline 0.80 & 1.084 & 495.40 & 7.66 & 40.80 & 15.76 & 79.02 & 5.01 \\
\hline 0.90 & 1.167 & 697.52 & 10.54 & 55.41 & 26.30 & 134.43 & 5.11 \\
\hline 1.00 & 1.290 & 1084.00 & & & & & \\
\hline
\end{tabular}

TABLE 12: SUE link flows and degrees of saturations for $\alpha$ values $0.0,0.8$ and 1.0.

\begin{tabular}{|c|c|c|c|c|c|c|c|c|}
\hline & $\alpha=0.0$ & & & $\alpha=0.8$ & & & $\alpha=1.0$ & \\
\hline Link Number & $\begin{array}{l}\text { SUE flows } \\
\text { (veh/h) }\end{array}$ & $\begin{array}{c}\text { Degree of } \\
\text { Saturation } \\
(\%)\end{array}$ & Link Number & $\begin{array}{l}\text { SUE flows } \\
\text { (veh/h) }\end{array}$ & $\begin{array}{c}\text { Degree of } \\
\text { Saturation } \\
(\%)\end{array}$ & Link Number & $\begin{array}{l}\text { SUE flows } \\
\text { (veh/h) }\end{array}$ & $\begin{array}{c}\text { Degree of } \\
\text { Saturation } \\
(\%)\end{array}$ \\
\hline 1 & 722 & 39 & 1 & 780 & 42 & 1 & 927 & 50 \\
\hline 2 & 458 & 36 & 2 & 500 & 55 & 2 & 595 & 61 \\
\hline 3 & 722 & 36 & 3 & 780 & 45 & 3 & 927 & 64 \\
\hline 4 & 596 & 47 & 4 & 643 & 52 & 4 & 765 & 72 \\
\hline 5 & 641 & 53 & 5 & 692 & 63 & 5 & 822 & 76 \\
\hline 6 & 175 & 32 & 6 & 190 & 35 & 6 & 226 & 45 \\
\hline 7 & 458 & 91 & 7 & 500 & 78 & 7 & 595 & 76 \\
\hline 8 & 474 & 64 & 8 & 517 & 70 & 8 & 616 & 72 \\
\hline 9 & 109 & 74 & 9 & 119 & 54 & 9 & 142 & 89 \\
\hline 10 & 474 & 92 & 10 & 517 & 83 & 10 & 616 & 93 \\
\hline 11 & 497 & 68 & 11 & 542 & 87 & 11 & 639 & 98 \\
\hline 12 & 253 & 23 & 12 & 271 & 25 & 12 & 329 & 29 \\
\hline 13 & 450 & 68 & 13 & 488 & 75 & 13 & 581 & 97 \\
\hline 14 & 785 & 62 & 14 & 858 & 70 & 14 & 1011 & 95 \\
\hline 15 & 786 & 48 & 15 & 859 & 61 & 15 & 1013 & 86 \\
\hline 16 & 386 & 95 & 16 & 719 & 68 & 16 & 847 & 91 \\
\hline 17 & 415 & 78 & 17 & 443 & 97 & 17 & 537 & 86 \\
\hline 18 & 354 & 74 & 18 & 377 & 63 & 18 & 457 & 62 \\
\hline 19 & 904 & 76 & 19 & 679 & 80 & 19 & 817 & 89 \\
\hline 20 & 1290 & 85 & 20 & 1399 & 92 & 20 & 1664 & 99 \\
\hline 21 & 1070 & 67 & 21 & 1160 & 74 & 21 & 1379 & 98 \\
\hline 22 & 1250 & 53 & 22 & 1355 & 66 & 22 & 1613 & 90 \\
\hline 23 & 574 & 59 & 23 & 917 & 75 & 23 & 1091 & 71 \\
\hline
\end{tabular}


TABLE 13: Values of signal timings, $\mu$ and PI for $\alpha$ values $0.0,0.8$ and 1.0.

\begin{tabular}{|c|c|c|c|c|c|c|c|c|}
\hline & & & & & Du & ion of sta & (s) & \\
\hline & O-D multiplier & Performance index & Cycle Time & Junction Number & Stage 1 & Stage 2 & Stage 3 & Offsets (s) \\
\hline & $\mu$ & $P I$ & $c(s)$ & $j$ & $\varphi_{i, 1}$ & $\varphi_{i, 1}$ & $\varphi_{i, 1}$ & $\theta_{i}$ \\
\hline & & & & 1 & 26 & 124 & --- & 0 \\
\hline & & & & 2 & 99 & 51 & --- & 0 \\
\hline$\alpha=0.0$ & 1.00 & 341.10 & 150 & 3 & 86 & 64 & --- & 79 \\
\hline & & & & 4 & 60 & 40 & 50 & 120 \\
\hline & & & & 5 & 18 & 52 & 80 & 0 \\
\hline & & & & 6 & 47 & 103 & --- & 149 \\
\hline & & & & 1 & 55 & 83 & --- & 0 \\
\hline & & & & 2 & 80 & 58 & --- & 50 \\
\hline$\alpha=0.8$ & 1.08 & 495.40 & 138 & 3 & 80 & 58 & --- & 74 \\
\hline & & & & 4 & 48 & 44 & 46 & 62 \\
\hline & & & & 5 & 23 & 42 & 73 & 89 \\
\hline & & & & 6 & 54 & 84 & --- & 61 \\
\hline & & & & 1 & 53 & 97 & --- & 0 \\
\hline & & & & 2 & 73 & 77 & --- & 2 \\
\hline$\alpha=1.0$ & 1.29 & 1084.00 & 150 & 3 & 95 & 55 & --- & 100 \\
\hline$a-1.0$ & & & & 4 & 54 & 50 & 46 & 0 \\
\hline & & & & 5 & 19 & 60 & 71 & 16 \\
\hline & & & & 6 & 70 & 80 & --- & 72 \\
\hline
\end{tabular}

provides a balance between conflicting issues, namely, reserve capacity maximization and delay minimization. Secondly, the proposed model is applied to Allsop \& Charlesworth's road network in order to show its effectiveness on a medium-sized signalized road network. According to the Pareto efficiency analysis for this network, it is found that the case where $\alpha$ equals 0.8 is also suitable since all other Pareto solutions proportionally reduce users' benefit more than the gain in network reserve capacity. Moreover, results indicate that there are no oversaturated links in the network even in the case where $\alpha$ equals 1.0 , which means that the $\mathrm{O}-\mathrm{D}$ multiplier is maximized.

In conclusion, results show that the use of different $\alpha$ values gives an opportunity to responsible authorities to make a suitable choice for the benefit of road users between two conflicting issues. For future studies, dynamic traffic assignment rather than static assignment may be considered in order to reflect the effect of time-varying flows on the solution of the proposed model.

\section{Data Availability}

The data used to support the findings of this study are included within the article.

\section{Conflicts of Interest}

The authors declare that there is no conflict of interest regarding the publication of this paper.

\section{References}

[1] F. V. Webster and B. M. Cobbe, "Traffic signal," Road Research Technical Paper 56, HMSO, London, UK, 1966.

[2] R. E. Allsop, "Estimating the traffic capacity of a signalized road junction," Transportation Research, vol. 6, no. 3, pp. 245-255, 1972.

[3] S. Yagar, "Capacity of a signalized road junction: critique and extensions," Transportation Research, vol. 8, no. 2, pp. 137-147, 1974.

[4] S. Yagar, "Addressing errors and omissions in paper on intersection capacity maximization," Transportation Research Part B: Methodological, vol. 19, no. 1, pp. 81-84, 1985.

[5] S. C. Wong and H. Yang, "Reserve capacity of a signal-controlled road network," Transportation Research Part B: Methodological, vol. 31, no. 5, pp. 397-402, 1997.

[6] H. Yang, M. G. H. Bell, and Q. Meng, "Modeling the capacity and level of service of urban transportation networks," Transportation Research Part B: Methodological, vol. 34, no. 4, pp. 255-275, 2000.

[7] H. Yang and J. Y. Wang, "Travel time minimization versus reserve capacity maximization in the network design problem," Transportation Research Record, vol. 1783, no. 1, pp. 17-26, 2002.

[8] G. Ziyou and S. Yifan, "A reserve capacity model of optimal signal control with user-equilibrium route choice," Transportation Research Part B: Methodological, vol. 36, no. 4, pp. 313-323, 2002.

[9] Y. E. Ge, H. M. Zhang, and W. H. Lam, "Network reserve capacity under influence of traveler information," Journal of Transportation Engineering, vol. 129, no. 3, pp. 262-270, 2003.

[10] H. Ceylan and M. G. H. Bell, "Reserve capacity for a road network under optimized fixed time traffic signal control," Journal 
of Intelligent Transportation Systems: Technology, Planning, and Operations, vol. 8, no. 2, pp. 87-99, 2004.

[11] A. Chen, P. Chootinan, and S. Wong, "New reserve capacity model of signal-controlled road network," Transportation Research Record, vol. 1964, pp. 35-41, 2006.

[12] S.-W. Chiou, "Reserve capacity of signal-controlled road network," Applied Mathematics and Computation, vol. 190, no. 2, pp. 1602-1611, 2007.

[13] S. Chiou, "An efficient search algorithm for road network optimization," Applied Mathematics and Computation, vol. 201, no. 1-2, pp. 128-137, 2008.

[14] S. Chiou, "Optimization of limited network capacity with toll settings," Information Sciences, vol. 179, no. 1-2, pp. 109-119, 2009.

[15] S. Chiou, "A hybrid approach for optimal design of signalized road network," Applied Mathematical Modelling, vol. 32, no. 2, pp. 195-207, 2008.

[16] S. Chiou, "Optimization for signal setting problems using nonsmooth techniques," Information Sciences, vol. 179, no. 17, pp. 2985-2996, 2009.

[17] E. Miandoabchi and R. Z. Farahani, "Optimizing reserve capacity of urban road networks in a discrete Network Design Problem," Advances in Engineering Software, vol. 42, no. 12, pp. 1041-1050, 2011.

[18] E. Miandoabchi, F. Daneshzand, W. Y. Szeto, and R. Zanjirani Farahani, "Multi-objective discrete urban road network design," Computers \& Operations Research, vol. 40, no. 10, pp. 24292449, 2013.

[19] S. Chiou, "Optimal signal-setting for road network with maximum capacity," Information Sciences, vol. 273, pp. 287-303, 2014.

[20] J. Wang, W. Deng, and J. Zhao, "Road network reserve capacity with stochastic user equilibrium," Transport, vol. 30, no. 1, pp. 103-116, 2015.

[21] H. Xiao, J. Gao, and Z. Zou, "Reserve capacity model based on variable demand for land-use development control," Transportation Planning and Technology, vol. 40, no. 2, pp. 199-212, 2016.

[22] F. Han and L. Cheng, "Stochastic user equilibrium model with a tradable credit scheme and application in maximizing network reserve capacity," Engineering Optimization, vol. 49, no. 4, pp. 549-564, 2016.

[23] O. Baskan and C. Ozan, "Reserve capacity model for optimizing traffic signal timings with an equity constraint," in Highway Engineering, IntechOpen, 2017.

[24] X. Li and J. Sun, "Multi-objective optimal predictive control of signals in urban traffic network," Journal of Intelligent Transportation Systems: Technology, Planning, and Operations, pp. 119, 2019.

[25] McTrans Center, "TRANSYT-7F Release 11.3 Users Guide," University of Florida, Gaineville, Florida, USA, 2008.

[26] F. V. Webster, “Traffic signal settings," Road Research Technical Paper 39, HMSO, London, UK, 1958.

[27] R. E. Allsop, "Delay-minimizing settings for fixed-time traffic signals at a single road junction," Journal of the Institute of Mathematics and Its Applications, vol. 8, pp. 164-185, 1971.

[28] S. Wong, "Derivatives of the performance index for the traffic model from TRANSYT," Transportation Research Part B: Methodological, vol. 29, no. 5, pp. 303-327, 1995.

[29] B. Heydecker, "A decomposition approach for signal optimisation in road networks," Transportation Research Part B: Methodological, vol. 30, no. 2, pp. 99-114, 1996.
[30] S. C. Wong, "Group-based optimisation of signal timings using the transyt traffic model," Transportation Research Part B: Methodological, vol. 30, no. 3, pp. 217-244, 1996.

[31] S. C. Wong, "Group-based optimisation of signal timings using parallel computing," Transportation Research Part C: Emerging Technologies, vol. 5, no. 2, pp. 123-139, 1997.

[32] S. C. Wong, W. T. Wong, C. M. Leung, and C. O. Tong, "Group-based optimization of a time-dependent TRANSYT traffic model for area traffic control," Transportation Research Part B: Methodological, vol. 36, no. 4, pp. 291-312, 2002.

[33] M. Girianna and R. F. Benekohal, "Application of genetic algorithms to generate optimum signal coordination for congested networks," in Proceedings of the 7th International Conference on Applications of Advanced Technology in Transportation, pp. 762$769,2002$.

[34] H. Ceylan and M. G. H. Bell, "Traffic signal timing optimisation based on genetic algorithm approach, including drivers' routing," Transportation Research Part B: Methodological, vol. 38, no. 4, pp. 329-342, 2004.

[35] H. Ceylan and M. G. Bell, "Genetic algorithm solution for the stochastic equilibrium transportation networks under congestion," Transportation Research Part B: Methodological, vol. 39, no. 2, pp. 169-185, 2005.

[36] H. Ceylan, "Developing combined genetic algorithm hillclimbing optimization method for area traffic control," Journal of Transportation Engineering, vol. 132, no. 8, pp. 663-671, 2006.

[37] J. Chen and L. Xu, "Road-junction traffic signal timing optimization by an adaptive particle swarm algorithm," in Proceedings of the 9th International Conference on Control, Automation, Robotics and Vision, vol. 1-5, pp. 1103-1109, 2006.

[38] C. Dan and G. Xiaohong, "Study on intelligent control of traffic signal of urban area and microscopic simulation," in Proceedings of the 8th International Conference of Chinese Logistics and Transportation Professionals, Logistics: The Emerging Frontiers of Transportation and Development in China, pp. 4597-4604, 2008.

[39] Z. Li, "Modeling arterial signal optimization with enhanced cell transmission formulations," Journal of Transportation Engineering, vol. 137, no. 7, pp. 445-454, 2011.

[40] Y. Liu and G.-L. Chang, "An arterial signal optimization model for intersections experiencing queue spillback and lane blockage," Transportation Research Part C: Emerging Technologies, vol. 19, no. 1, pp. 130-144, 2011.

[41] H. Ceylan, "A hybrid harmony search and TRANSYT hill climbing algorithm for signalized stochastic equilibrium transportation networks," Transportation Research Part C: Emerging Technologies, vol. 25, pp. 152-167, 2012.

[42] M. Dell'Orco, O. Baskan, and M. Marinelli, "A Harmony Search Algorithm approach for optimizing traffic signal timings," PROMET - Traffic\&Transportation, vol. 25, no. 4, pp. 349-358, 2013.

[43] M. Dell’Orco, Ö. Başkan, and M. Marinelli, "Artificial bee colony-based algorithm for optimising traffic signal timings," in Soft Computing in Industrial Applications, Advances in Intelligent Systems and Computing, V. Snášel, P. Krömer, M. Köppen, and G. Schaefer, Eds., vol. 223, pp. 327-337, Springer, Berlin, Germany, 2014.

[44] F. Zhu, H. A. Aziz, X. Qian, and S. V. Ukkusuri, "A junctiontree based learning algorithm to optimize network wide traffic control: a coordinated multi-agent framework," Transportation Research Part C: Emerging Technologies, vol. 58, pp. 487-501, 2015. 
[45] C. Ozan, O. Baskan, S. Haldenbilen, and H. Ceylan, "A modified reinforcement learning algorithm for solving coordinated signalized networks," Transportation Research Part C: Emerging Technologies, vol. 54, pp. 40-55, 2015.

[46] E. Christofa, K. Ampountolas, and A. Skabardonis, "Arterial traffic signal optimization: a person-based approach," Transportation Research Part C: Emerging Technologies, vol. 66, pp. 27-47, 2016.

[47] L. Zhang, Z. Song, X. Tang, and D. Wang, "Signal coordination models for long arterials and grid networks," Transportation Research Part C: Emerging Technologies, vol. 71, pp. 215-230, 2016.

[48] S. Srivastava and S. K. Sahana, "Nested hybrid evolutionary model for traffic signal optimization," Applied Intelligence, vol. 46, no. 1, pp. 113-123, 2017.

[49] S. Memoli, G. E. Cantarella, S. de Luca, and R. D. Pace, "Network signal setting design with stage sequence optimisation," Transportation Research Part B: Methodological, vol. 100, pp. 20-42, 2017.

[50] M. G. H. Bell and Y. Iida, Transportation Network Analysis, John Wiley and Sons, Chichester, UK, 1997.

[51] M. G. H. Bell and C. M. Shield, "A log-linear model for path flow estimation," in Proceedings of the 4th International Conference on the Applications of Advanced Technologies in Transportation Engineering, Y. J. Stephanedes and F. Filippi, Eds., pp. 695-699, Capri, Italy, 1995.

[52] Z.-C. Li and X.-Y. Ge, "Traffic signal timing problems with environmental and equity considerations," Journal of Advanced Transportation, vol. 48, no. 8, pp. 1066-1086, 2014.

[53] E. Ulungu, J. Teghem, P. Fortemps, and D. Tuyttens, "MOSA method: a tool for solving multiobjective combinatorial optimization problems," Journal of Multi-Criteria Decision Analysis, vol. 8, no. 4, pp. 221-236, 1999.

[54] R. Storn and K. Price, "Differential evolution-a simple and efficient heuristic for global optimization over continuous spaces," Journal of Global Optimization, vol. 11, no. 4, pp. 341359, 1997.

[55] L. Nolle, I. Zelinka, A. Hopgood, and A. Goodyear, "Comparison of an self-organizing migration algorithm with simulated annealing and differential evolution for automated waveform tuning," Advances in Engineering Software, vol. 36, no. 10, pp. 645-653, 2005.

[56] L. Vincenzi, G. De Roeck, and M. Savoia, "Comparison between coupled local minimizers method and differential evolution algorithm in dynamic damage detection problems," Advances in Engineering Software, vol. 65, pp. 90-100, 2013.

[57] P. Civicioglu and E. Besdok, "A conceptual comparison of the Cuckoo-search, particle swarm optimization, differential evolution and artificial bee colony algorithms," Artificial Intelligence Review, vol. 39, no. 4, pp. 315-346, 2013.

[58] A. Deb, J. S. Roy, and B. Gupta, "Performance comparison of differential evolution, particle swarm optimization and genetic algorithm in the design of circularly polarized microstrip antennas," IEEE Transactions on Antennas and Propagation, vol. 62, no. 8, pp. 3920-3928, 2014.

[59] P. Bracinik, K. Dezelak, A. Otcenasova, and M. Höger, "Comparison between the particle swarm optimisation and differential evolution approaches for the optimal proportional-integral controllers design during photovoltaic power plants modelling," IET Renewable Power Generation, vol. 10, no. 4, pp. 522-530, 2016.
[60] R. E. Allsop and J. A. Charlesworth, "Traffic in a signalcontrolled road network: an example of different signal timings inducing different routeings," Traffic Engineering \& Control, vol. 18, no. 5, pp. 262-265, 1977.

[61] H. Ceylan, A genetic algorithm approach to the equilibrium network design problem [Ph.D. Thesis], University of Newcastle upon Tyne, Newcastle, UK, 2002.

[62] J. Ronkkonen, V. Kukkonen, and K. V. Price, "Real-parameter optimization with differential evolution," in Proceedings of the IEEE Congress on Evolutionary Computation, pp. 506-513, Edinburgh, Scotland, September 2005. 


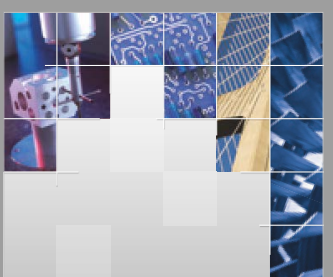

\section{Enfincering}
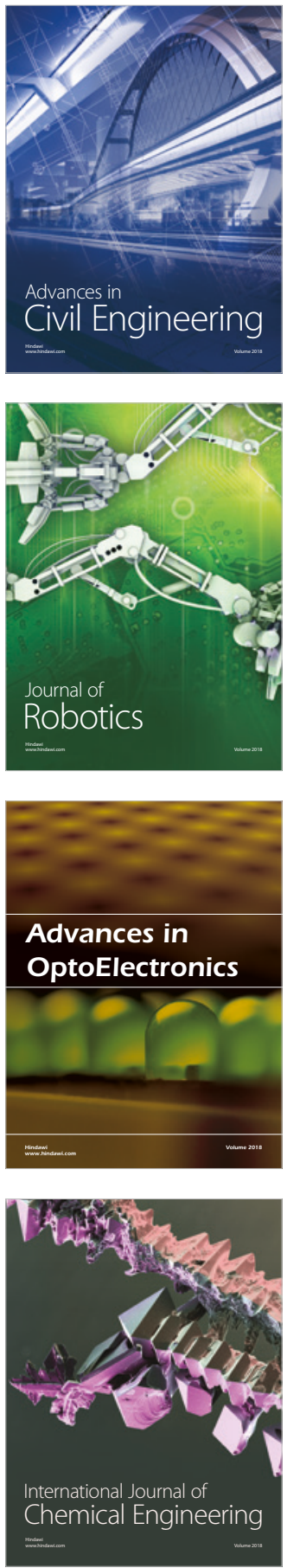

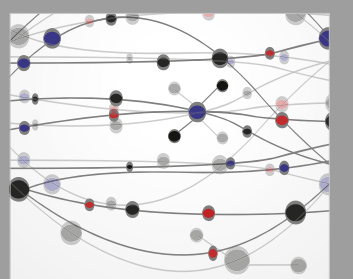

\section{Rotating \\ Machinery}

The Scientific World Journal

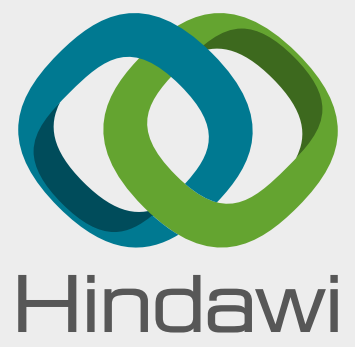

Submit your manuscripts at

www.hindawi.com
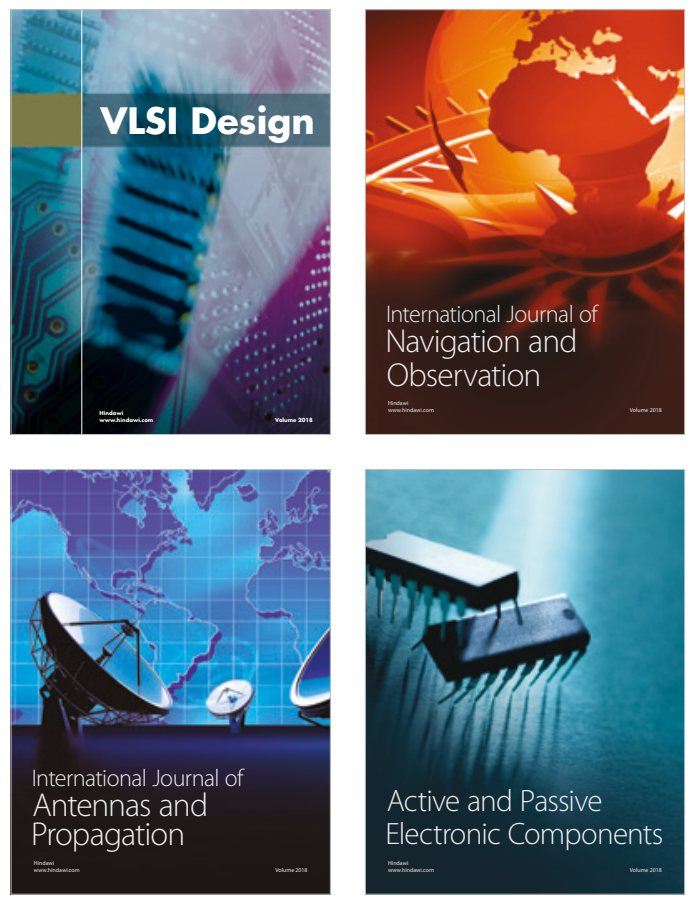
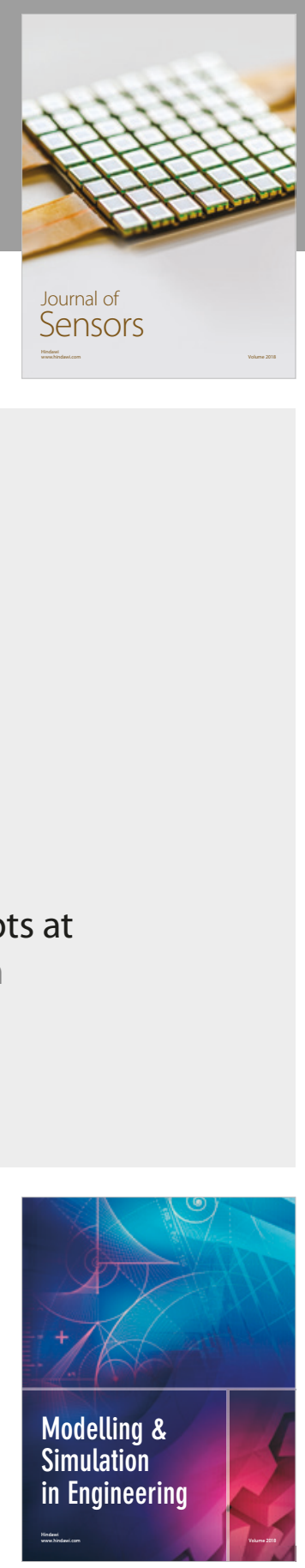

\section{Advances \\ Multimedia}
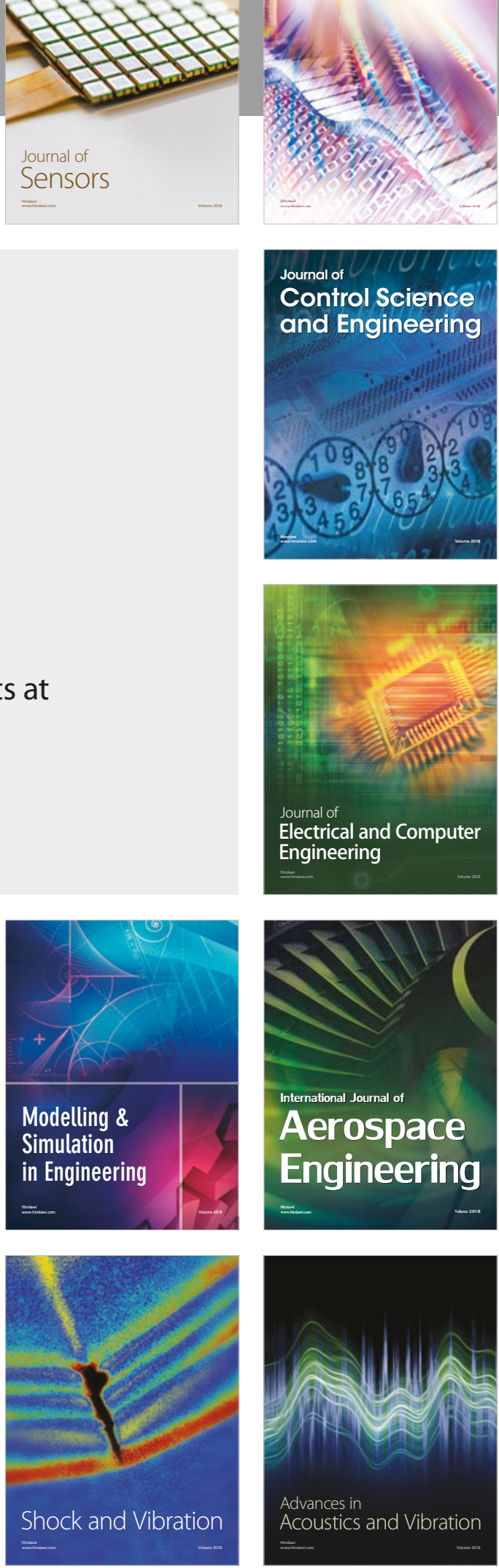